\title{
Zur Fortpflanzungsbiologie der Naticiden (Gastropoda Prosobranchia)
}

(Laichringe und Laichakt bei Lunatia nitida Donovan) ${ }^{1}$ )

\author{
Von Erich Ziegelmeier \\ Aus der Biologischen Anstalt Helgoland
}

(Mit 13 Abbildungen und 3 Tabellen)

Inhaltsübersicht

I. Einleitung S.94 - II. Vorkommen von Lunatia nitida im deutschen Nordseegebiet S. 95 III. Laichringe von Lunatia nitida S. 97 - a) Form. Struktur, Größe und Windungen S. 97 b) Eiverteilung, -zahlen, -größe S. 102 - IV. Laichakt im Aquarium S. 103 - a) Geschlechtlichkeit bei Lunatia nitida S. 103 - b) Fruchtbarkeit der nitida- 99 und Laichzeiten S. 107 c) Direkte Beobachtung der Laichablage S. 108 - V. Kurze Bemerkungen über die ersten Furchungsstadien und das Schlüpfen der Veligerlarven S. 114 - VI. Zusammenfassung S. 116 VII. Literaturverzeichnis S. 117.

\section{Einleitung}

Im Verlaufe der Untersuchungen über den Bau und die Funktion des von Schiemenz 1891 bei den Naticidae als "Bohrdrüse" bezeichneten tellerförmigen Organs am Vorderende des Rüssels hinter der Mundöffnung ergab sich die Notwendigkeit, neben der Anwendung verschiedener Verfahren, auch die Entwicklung dieses Drüsenpilzes bei den einzelnen Larvenstadien und während der Metamorphose histologisch zu verfolgen.

Daß die "Bohrdrüse" nicht im Sinne von SchiEmenz chemisch an der Herstellung der dem Nahrungserwerb dienenden Löcher in den Schalen lebender Muscheln und Schnecken beteiligt ist, sondern die Naticiden mechanisch mit der Radula bohren, konnte bei der kleinen, in der Deutschen Bucht häufigsten Nabelschnecke Lunatia nitida nachgewiesen (ZiEgELMEIER 1954) und bei Untersuchungen an Lunatia catena und den mediterranen Arten Natica hebraea. $\mathcal{N}$. millepunctata sowie Polynices jasephinus bestätigt werden (noch unveröffentlicht). Die eigentliche Funktion der "Bohrdrüse" sollen die z. Zt. laufenden Versuche klären.

Während der Teiluntersuchungen, die sich zum Zwecke der Beschaffung von Larvenmaterial mit der Entwicklungsgeschichte von Lunatia nitida befaßten, konnte eine Reihe von Beobachtungen zur Erweiterung der Kenntnis über den Laich dieser Nabelschnecke beitragen und die Art und Weise der Laichabgabe klären.

Die von den Naticiden abgelegten Laichprodukte gaben wegen ihrer eigenartigen Form häufig Anlaßs zu Untersuchungen und erhielten so von den einzelnen Autoren die verschiedensten Bezeichnungen: "egg mass" (Thorson 1946, Amo 1955) - „egg collar“" (Giglioli 1955) - „sand collar" (Morris 1950, Mc Gintrie 1949) - „sandy ribbon“ (Eales 1950) — „egg aidus“ (Dell 1956) - „egg ribbon“ (Natarajan 1957) - , eggs in sandy spirals“ oder "very much flattened, usually incomplete, slightly spiral ring " (LEBour 1936) - „eiersnoer" (van Benthem-Jutting 1933) — „Laichband“ (Ankel 1936, Hertling 1932).

1) Herrn Prof. Dr. A. Bückmann zum 60. Geburtstag gewidmet. 
Die Laichgelege von Lunatia nitida - ihrer Form wegen hier "Laichringe" genannt - sind von HerTLuNg in einer kurzen Darstellung beschrieben und zum erstenmal abgebildet worden. Leider stützten sich die dort mitgeteilten Feststellungen auf nur wenige Beobachtungen; die Beschreibung der Laichringe ist nicht befriedigend, in einigen Punkten unklar. Es ist daher notwendig, verschiedenes zu ergänzen und z. T. zu berichtigen, worauf in den entsprechenden Abschnitten näher eingegangen wird. Weitere Beschreibungen und Abbildungen des Laichringes und der postembryonalen Entwicklungsstadien von Lunatia nitida bei Ankel 1936, Marie V. Lebour und Thorson.

Eine Zusammenstellung von Laichgelegen verschiedener Arten der Naticidae gibt GiglıoLI, der auch erstmalig über den Vorgang der Laichabgabe bei den im Eulitoral lebenden Polynices triseriata und P. heros berichtet. Der von Giglioli geschilderte Laichakt bei den erwähnten Naticidenarten deckt sich jedoch nicht mit den Beobachtungen über die Art und Weise, mit der L. nitida die Laichringe formt.

\section{Vorkommen von Lunatia nitida im deutschen Nordseegebiet}

Die beiden in der südlichen Nordsee häufiger vorkommenden Arten der Naticidengattung Lunatia sind L. catena und L. nitida. Während die bis über $40 \mathrm{~mm}$ Schalenhöhe erreichende L. catena in unseren Meeresgebieten die küstenferneren, sandigen Gründe bewohnt, ist die kleinere L. nitida (bis $18 \mathrm{~mm}$ ) auch in küstennäheren Gewässern anzutreffen und bevorzugt hier besonders die schlicksandigen Böden.

Nach quantitativen und qualitativen Bodenfaunauntersuchungen im Ostteil der Deutschen Bucht in der Zeit von Herbst 1949 bis Frühjahr 1960 ergeben sich bezüglich des Vorkommens von Lunatia und deren Abhängigkeit von der Bodenbeschaffenheit folgende Feststellungen:

Die L. nitida-Bestände schwanken in den verschiedenen Jahren mit der Abundanz der Muscheln, den Hauptnahrungstieren der Nabelschnecken. Bei geringem Nahrungsangebot ist die kleine Nabelschnecke im Untersuchungsgebiet nur auf 3 Stationen bei Helgoland (Nordhafen innen, Alter Fischplatz außen und Nordhafen außen) zu finden (Abb. 1), und zwar in schillhaltigen, grobsandigen Schlickböden oder in sandigem, fast zähem Schlick bei Tiefen von $30-40 \mathrm{~m}$.

Eine Massenentwicklung von Lamellibranchiern hat stets eine dichtere Besiedlung von L. nitida zur Folge; die Anzahl der Stationen, auf denen die Schnecke anzutreffen ist, wird größer (Abb. 2). Sedimente mit reichlicherem Sandanteil, in denen vorher keine L. nitida zu finden waren, sind jetzt bewohnt. Unbesiedelt jedoch bleiben, wegen der durch die starken Strömungen bedingten ständigen Sedimentumlagerungen, die flacheren um $10 \mathrm{~m}$ tiefen Reinsandgründe.

Die Sandverdriftungen bilden vor allem eine Gefahr für die von den Schnecken abgelegten Laichringe, die, nicht wie Kristensen 1959 angibt, für einige Zeit im Sand liegenbleiben, sondern unmittelbar nach dem Laichakt vom Lunatia- 9 selbst auf die Sedimentoberfläche transportiert werden. Außerhalb des Sandes im freien Wasser werden den Eiern im Laichring die notwendigen Bedingungen für die Entwicklung geboten, wieder Einsanden des Laichgeleges in den strömungsreichen Gebieten würde sämtliche Larven vernichten. 


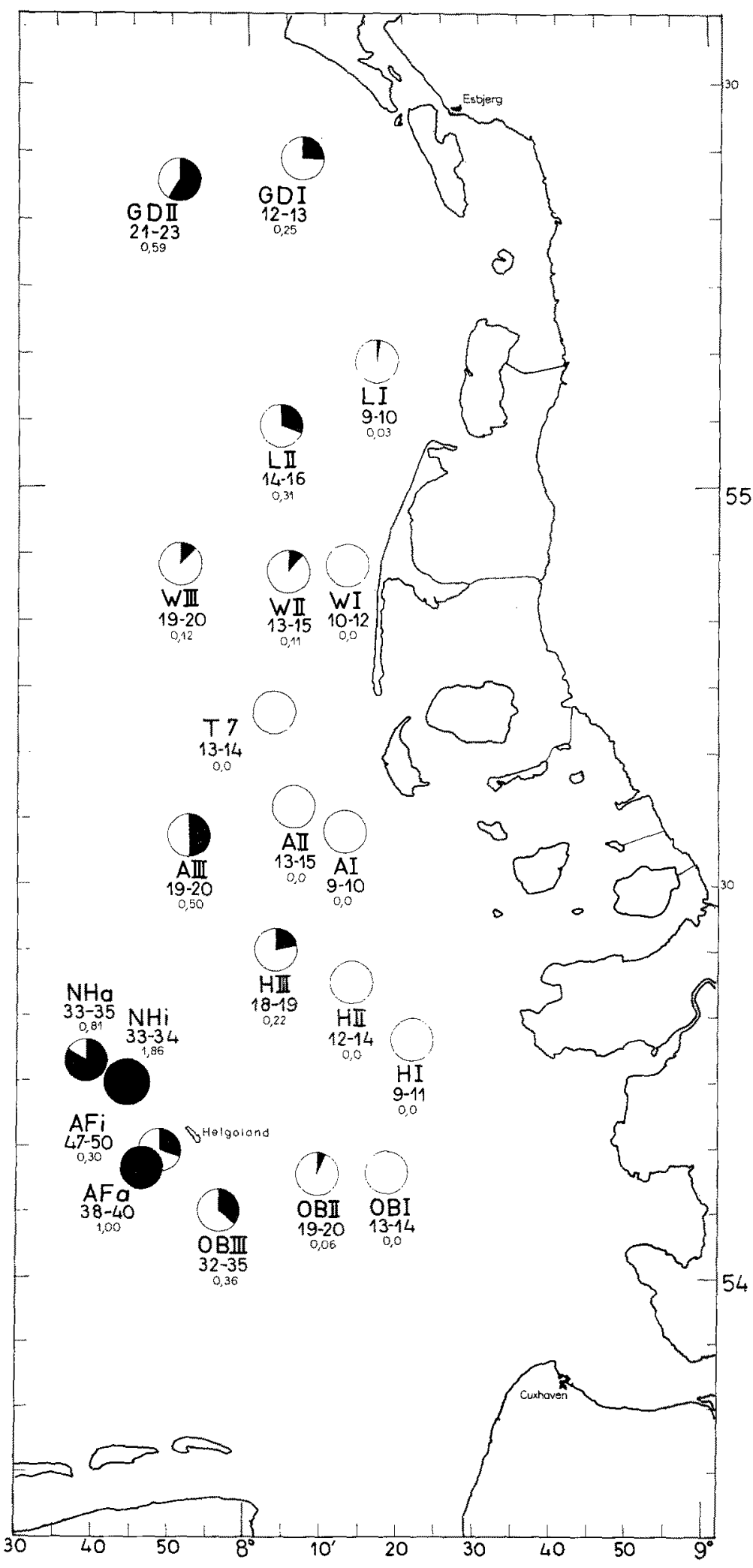




\section{Laichringe von Lunatia nitida}

a) Form, Struktur, Größe und Windungen

Das Laichprodukt von Lunatia nitida ist ein im Profil konisch aussehendes, wendelförmig übereinander liegendes Band, das aus vielen mit Schleim zusammengekitteten Sandkörnchen besteht, an und zwischen denen die Eier festgeheftet sind. Das aus einer oder mehreren Windungen bestehende Gelege stellt in der Aufsicht ein ringartiges Gebilde dar, womit die Bezeichnung „Laichring" ihre Begründung erhält.

In ihrer äußeren Gestalt und in der Anordnung der Eier nehmen die Laichringe von L. nitida innerhalb der Gelegeformen bei den Naticiden eine Sonderstellung ein. In den von Amio und Grglioli aufgestellten Einteilungen

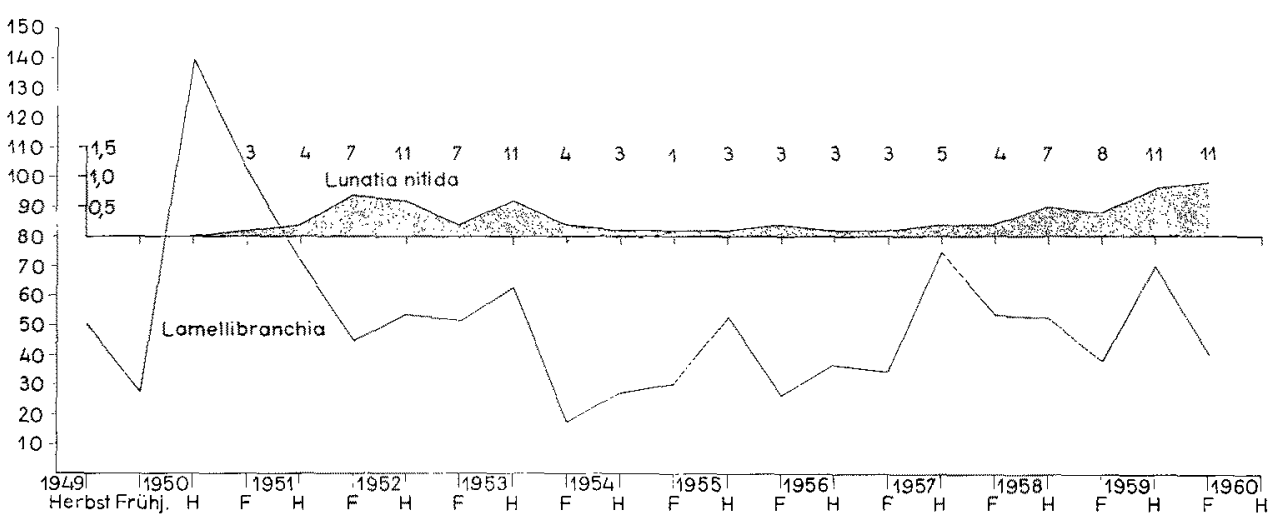

Abb. 2. Bestandszahlen von Muscheln und Lanatia nitida nach quantitativen und qualitativen Bodenfaunauntersuchungen im Ostteil der Deutschen Bucht für die Zeit von Herbst 1949 bis Frühjahr 1960.

Ordinate $=$ Anzahl auf $0,1 \mathrm{~m}^{2} \mathrm{im}$ Durchschnitt aus sämtlichen Stationen und Bodengreiferfängen aller Untersuchungsfahrten. Die obere Zahlenreibe gibt die Anzahl der Stationen an auf denen $L$. nitida in den verschiedenen Jahren und Jahreszeiten im Untersuchungsgebiet gefunden wurde.

läßt sich der nitida-Laich nicht einreihen. Am ehesten wäre nach der Darstellung einiger Naticiden-Gelege von THorson der Laich von Lunatia montagui, eine Nabelschneckenart, die in der Deutschen Bucht nicht vorkommt, mit den Laichringen von L. nitida vergleichbar. Das von ThORson abgebildete kurze Bruchstück eines L. montagui-Laichgeleges läßt vermuten, daß ebenso wie bei L. nitida die Ringe von L. montagui einen einfach nach außen gewölbten Querschnitt besitzen und nicht wie die meisten anderen bekannten Laichbänder von Naticiden im Profil entweder gerade Wände haben oder geschweift, d. h. in der unteren Hälfte konvex, im oberen Teil konkav sind (Abb. 3).

Abb, 1. Vorkommen von Lunatia nilida auf den Terminstationen der quantitativen und qualitativen Bodenfaunauntersuchungen im Ostteil der Deutschen Bucht. Die Zahlen unter den Stationsbezeichnungen geben die während der Zeit von Herbst 1949 bis Herbst 1959 bei den Probeentnahmen ermittelten Tiefen an; darunter die Abundanzgröße (schwarzer Kreissektor) $=$ durchschnittliche Bestandszahl der bei sämtlichen Untersuchungsfahrten aus den Bodengreifern der einzelnen Stationen gesammelten $L$. nitida umgerechnet auf $0,1 \mathrm{~m}^{2}$ für die gesamte Untersuchungszeit. 


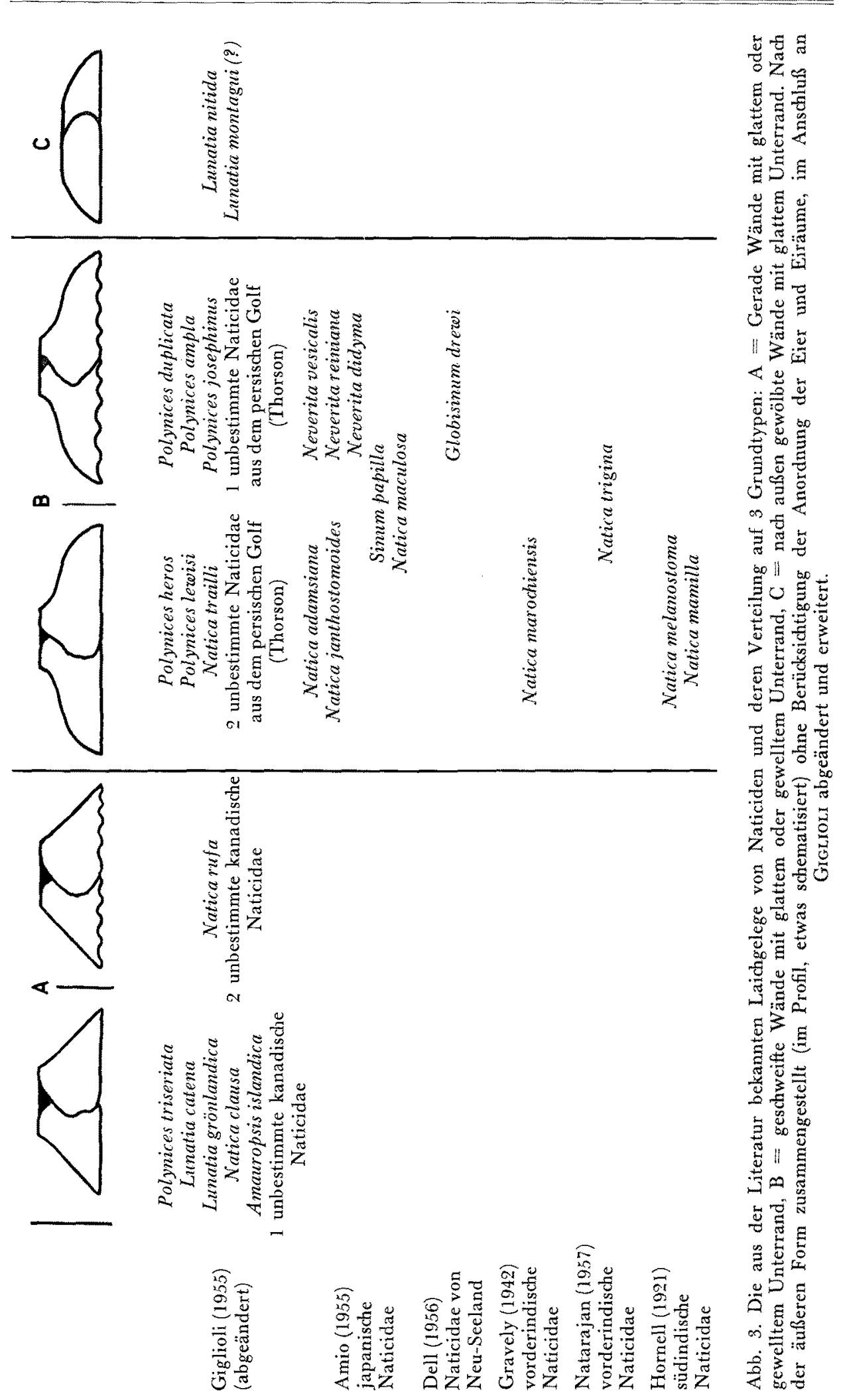



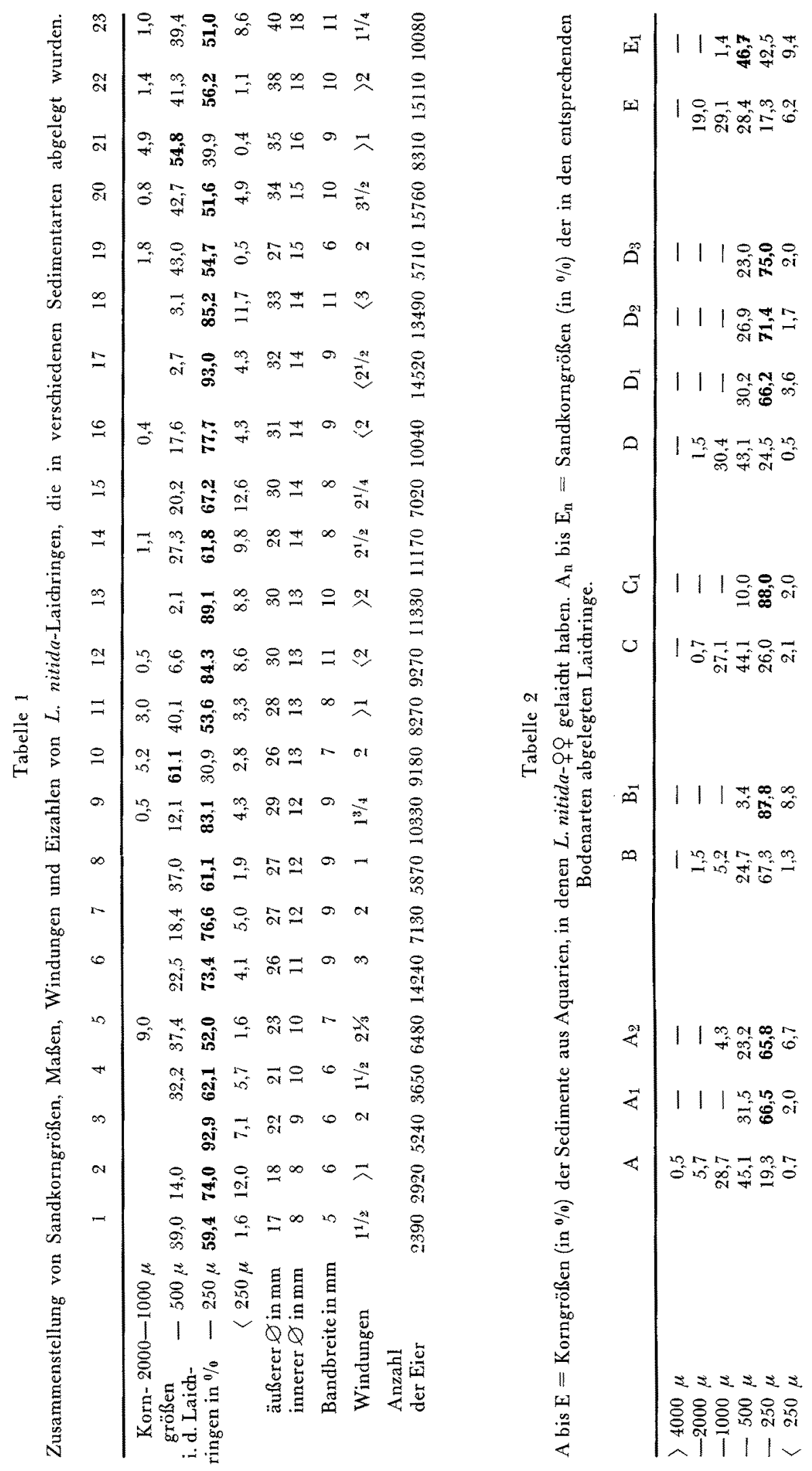
Die am häufigsten gefundenen Laichgelege der Naticiden gehören zum Typ B mit geschweiften Wänden und glattem oder gewelltem Unterrand. Bei den Zwischentypen handelt es sich um Nabelschnecken, bei denen innerhalb der gleichen Art Laichbänder abgelegt werden, die am unteren Rand glatt oder teilweise bzw. schwach bis stärker gewellt sein können.

Die von Giglioli vertretene Ansicht, daß bei den Laichformen der Gruppe B „owing to the lack of rigidity, the walls collapse and in profile assume a characteristic sigmoidal curvature", bedarf der Úberprüfung, denn nach Beobachtungen an Polynices josephinus, die hier im Aquarium häufig laichten, werden die Laichbänder vom $q$ bereits endgültig geformt und sinken nicht erst nach dem Laichakt etwas in sich zusammen, um die typische Gestalt zu erhalten.

Die nitida-Laichringe liegen bei einigermaßen planem Untergrund nicht wie Hertuing angibt „dem Sande flach auf“, sondern ruhen so auf dem Sediment, daß nur die untere Außenkante (.,basal margin“ nach Gigliour) den Boden berührt (Abb. 4). Der im Profil gemessene Neigungswinkel der nitidaLaichringe ist spitzer als bei den Laichbändern von Lunatia catena und den

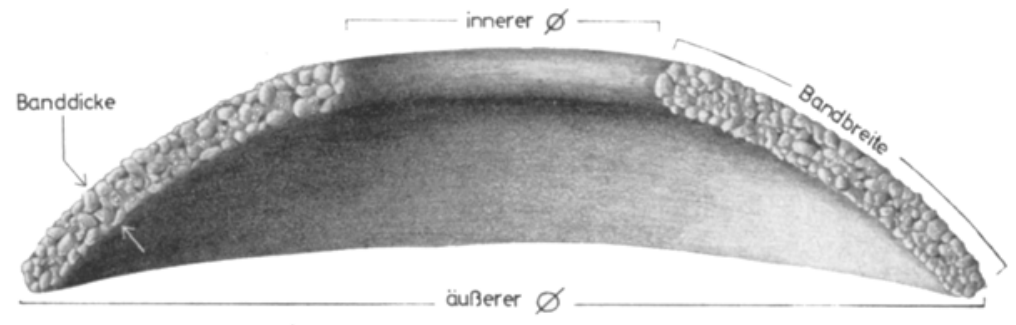

Abb. 4. Querschnitt durch einen Iaichring (1 Windung) von Lunatia nilida. halbschematisch. Die beiden Schnittflächen zeigen maßstabgetreu die Anordnung der Sandkörnchen und der Eier in den Eihüllen. Vergr. $3 x$.

anderen Natica-Arten. Die Wölbung des Laichringes von L. nitida - in Abb. 4 als die am häufigsten anzutreffende Form dargestellt — kann bei den einzelnen Gelegen auf Grund der Sedimentunterschiede variieren. Bei einigen Versuchen, bei denen die Weibchen in sehr feinem reinem Sand im Aquarium ihren Laich ablegen mußten, konnten fast plane Laichringe bcobachtet werden, die wegen ihrer weichen Konsistenz dann beinahe dem Sande auflagen. Vermutlich hat Hertung diese Ausnahmefälle verallgemeinert, bei denen die biegsamen Ringe nur dann zustande kommen, wenn Lunatia-qO in Sand mit sehr geringer Korngröße laichen. Bei der Laichringherstellung wird in diesen Fällen von der Schnecke mehr Schleim eingebaut, die im Ring verkitteten Sandkörnchen berühren sich kaum, und es fehlt ihnen im Verband die gegenseitige Stütze, wodurch das Gelege die meist zu beobachtende starre Form einbüßt.

Bemerkenswert ist die etwa gleiche Korngröße der Hauptmasse der von den Schnecken in die Ringe eingebauten Sandkörnchen. In der Tabelle 1 sind die Werte der Korngrößenanalysen ${ }^{2}$ ) von 23 nitida-Laichringen aus verschiedenen Bodenarten, deren Korngrößen jedoch nicht bestimmt werden konnten, zusammengestellt worden. Unabhängig von der Ringgröße liegen die Werte

פ) Herrn Dr. J. Jarke, Deutsches Hydrographisches Institut, Hamburg, herzlichen Dank für die Durchführung der Korngrößenbestimmungen. 
für die beim Ringbau verwandten Sandkorngrößen in den meisten Gelegen zwischen $250 \mu$ und $500 \mu$. Eine Gegenüberstellung von Korngrößen, die den Weibchen bei der Laichablage zur Verfügung standen und in den Ringen eingebaut waren, zeigt die Tabelle 2. Die Technik, mit der die Tiere während des Laichvorganges die etwa gleichgroßen Sandkörnchen einzubauen imstande sind, wird im Zusammenhang mit der Schilderung des Laichaktes beschrieben.
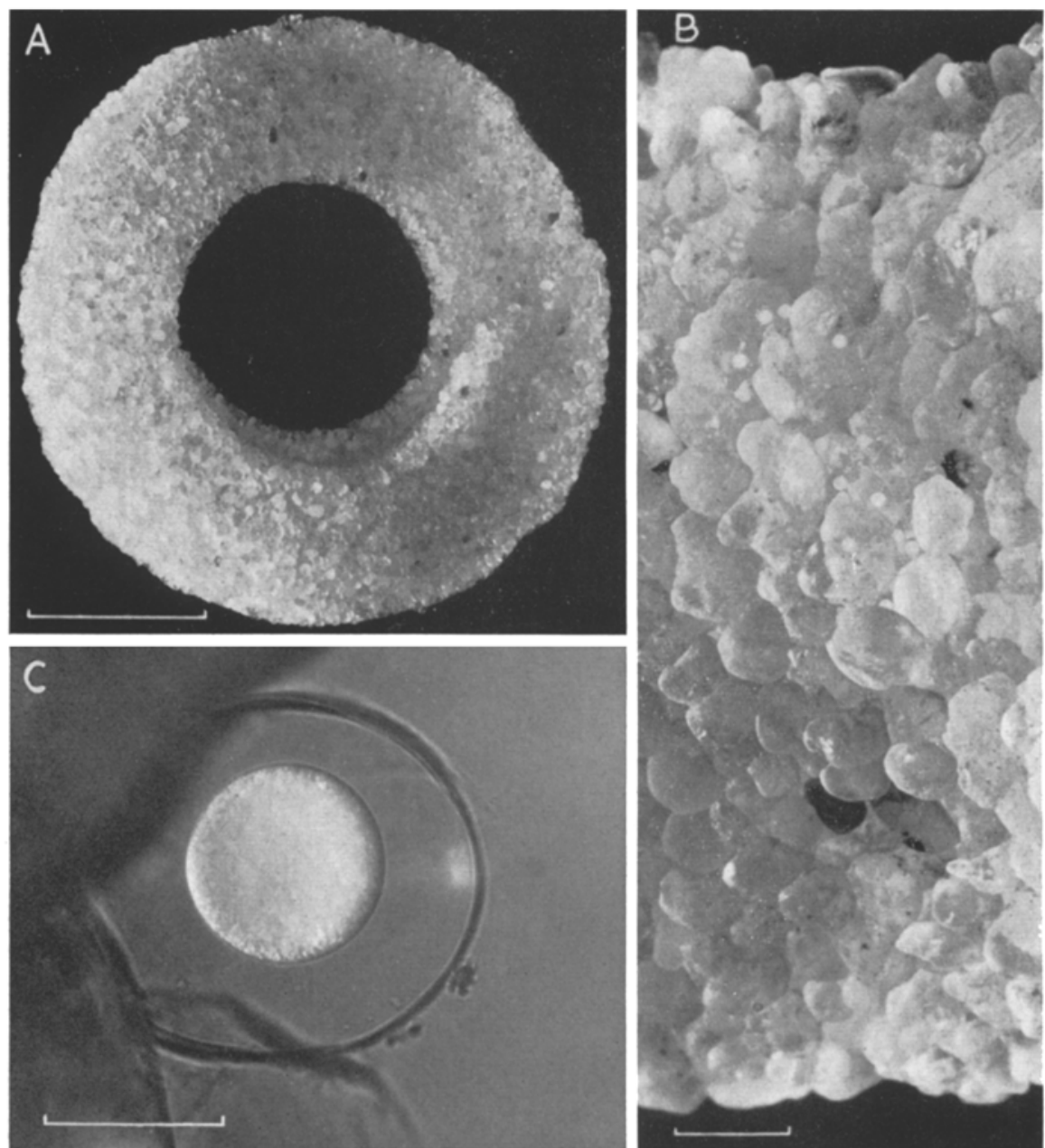

Abb. 5. $\mathrm{A}=L$, nitida-Laichring $\left(1 \frac{1}{2}\right.$ Windungen) von oben gesehen $(M a ß s t .=1 \mathrm{~cm})$, rechts das Ringende, $B=$ Ausschnittvergrößerung der Bandbreite eines Laichringes (Maßst. $=1 \mathrm{~mm}$ ) mit schwächerer Anhäufung von Eiern. $C=$ ungefurchtes $E i$ mit Hülle (Maßst. $=0,1 \mathrm{~mm}$ ).

Für die Größenangaben der nitida-Laichringe ist der innere $\phi$ das eindeutigste Maß, es entspricht der Gehäusehöhe des Lunatia-P. Diese Übereinstimmung wird bei der Darstellung des Laichvorganges verständlich. Die Bandbreite und damit der äußere $\phi$ der von demselben Tier abgelegten Ringe können variieren. Die Banddicke ist ebenso wie der Neigungswinkel der Laichringwand abhängig von der Art des Sediments: Je feiner der den Schnecken zur Verfügung stehende Sand ist, um so dünner wird das Band, und der Ring sinkt, wie oben bereits erwähnt, etwas mehr zusammen, der Nei- 
gungswinkel wird dadurch spitzer. Die Banddicke beträgt nach Messungen an zahlreichen Laichringen rd. 1 bis 2,5 mm. Dabei ist ein geringer Unterschied in der Dicke am oberen Rand gegenüber der am unteren festzustellen mit einer Differenz von etwa $0,3 \mathrm{~mm}$. Messungen der Neigungswinkel ergaben Werte von ca. $25^{\circ}-45^{\circ}$.

Die Anzahl der Windungen ist bei den einzelnen Laichringen verschieden. Nach Beobachtungen bei Schnecken, deren Laichen durchgehend im Aquarium verfolgt werden konnte, war festzustellen, daß gegen Ende der Laichperiode die Zahl der Windungen in den einzelnen Gelegen abnahm. Der letzte Ring bestand dann nur noch aus einer Windung. Maximal wurden $3^{1 / 2}$ Windungen gezählt. Auch hier besteht keine Abhängigkeit der Anzahl der Windungen von der Größe des Ringes (Tab. 1).

Betrachten wir einen nitida-Laichring von oben, so ergibt sich eine rechtsgewundene, im Uhrzeigersinn laufende Wendel (Abb. 5 A). In diesem $\mathrm{Zu}$ sammenhang sei bereits jetzt bemerkt, daß es sich bei dem unten liegenden Ende um den Anfang, bei dem oberen um den Abschluß des Ringes während des Laichaktes handelt.

b) Eiverteilung, -zahlen, -größe

Uber die Verteilung der Eier in den nitida-Laichringen berichtet HerTLING: „Die Außenflächen sind weit dichter mit sehr feinem Sand bedeckt,..., so daß man hier die einzelnen Eikammern nicht erkennen kann“. Diese Darstellung bedarf der Berichtigung. „Eikammern“, wie sie bei Laichbändern von Lunatia catena und bei den meisten anderen Naticidenarten bekannt sind, fehlen in den Laichringen von L. nitida. Auch darf hier nicht von eifreien „Außenflächen" gesprochen werden. Die dotterreichen Eier liegen in Eihüllen (Abb. 4 und 5), die an dem vom $q$ ausgeschiedenen, die Sandkörnchen zusammenhaltenden Mörtelschleim festgeheftet und über den gesamten Ring auf der Ober- sowie Unterseite und im Inneren verteilt sind, was nach Zerbrechen eines Ringes bei entsprechender Vergrößerung deutlich zu erkennen ist. Anhäufungen von Eiern an manchen Stellen des Laichringes können gelegentlich beobachtet werden (Abb. 5 B).

Angaben über die Anzahl von Eiern in Laichgelegen von Prosobranchiern sind in der älteren Literatur nicht sehr häufig. Von einigen Schneckenarten finden sich Eizahlen - in den zusammenfassenden Darstellungen z. T. von anderen Autoren übernommen - bei Simroth, Linke 1933, Ankel 1936, Portmann 1925, vor allem bei den Untersuchungen des letzteren über Nähreierbildung bei Buccinum und Nucella. Nähreier stellte Ankel 1930 auch in Laichbändern von Lunatia catena fest, bei denen von den in einer Eihöhle abgelegten 50 Eiern nur 3 bis 4 Embryonen aufkommen, der größte Teil der Eier ist Dotternahrung für die Larven.

In diesem Zusammenhang ist die von HerTLING gestellte Frage, "ob auch bei $\mathcal{N}$ atica pulchella“ (syn. Lunatia nitida) "Nähreier vorkommen oder fehlen" zu beantworten. Die Anordnung der im nitida-Laichring e in zeln und nicht, wie bei Lunatia catena und den meisten anderen Naticiden-Laichbändern in Eika mmern einzeln oder zu mehreren nebeneinander liegenden Eiern, schaltet die "Ernährung der Embryonen durch Nähreier" bei $L$. nitida aus.

Natarajan veröffentlicht in der Beschreibung der Laichgelege von 32 vorderindischen Prosobranchierarten aus 12 Familien auch die Eizahlen u. a. für Natica marochiensis $=2900$ und $\mathcal{N}$. tigrina $={ } 40-55$ thousand egg spaces in an egg-ribbon". 
Bei Lunatia nitida wurden bei Bouin- und Formalin-fixierten Laichringen Zählungen von ungefurchten Eiern, weiter entwickelten Stadien oder von den sich noch in den Eihüllen befindenden Larven durchgeführt. Zuvor ist jeder Ring in möglichst gleiche Abschnitte (ca. $10 \mathrm{~mm}$ ) zerlegt worden. Dabei ergab sich, daß die Eier, abgesehen von den bereits erwähnten gelegentlich auftretenden Anhäufungen an einzelnen Stellen, ziemlich gleichmäßig über den gesamten Laichring verteilt sind. Unterschiede in der Anzahl und in der Verteilung der Eier am Beginn und am Ende des Ringes waren nicht festzustellen, ebenso war keine Abhängigkeit der Eizahlen von der Größe und der Anzahl der Windungen zu erkennen. Die durchschnittliche Eizahl von 38 nitida-Laichringen (davon in Tab. $1=23$ ) beträgt 8140 Eier pro Ring (maximal $=15760$, minimal $=2390$ ).

Die Größe der sphärischen nitida-Eier geben Lebour und Thorson mit $0,160 \mathrm{~mm}$ an, die Eihülle mit $0,240-0,320 \mathrm{~mm}$. Nach den bei L. nitida an frisch abgelegten, ungefurchten, lebenden Eiern durchgeführten Messungen ergeben sich Werte, die unter den oben angeführten liegen. Der $\phi$ des nitidaEies beträgt $0,101-0,137 \mathrm{~mm}$, für die ellipsoidförmigen bis kugeligen Eihüllen konnten Werte von $0,208-0,254 \mathrm{~mm}$ ermittelt werden.

\section{Laichakt im Aquarium}

Wie bereits in der Einleitung erwähnt, ist die Art und Weise der Laichablage bei Naticiden von Giglon erstmalig für 2 Polynices-Arten beschrieben worden. Seine Beobachtungen an Polynices triseriata und P. heros im Watt konnten wegen der Gezeiten nicht permanent durchgeführt werden. Für die Darstellung des Laichvorganges blieb ihm daher nur die Möglichkeit, „to piece together individual observations made on the spawning snails and advance a detailed though partly hypothetical description of the manner of collar formation".

Zur Kenntnis des gesamten Laichvorganges sind Aquariumsuntersuchungen unbedingt erforderlich. Dabei wird vorausgesetzt, daß die von den Tieren im Aquarium angewandte Technik bei der Herstellung der Laidhringe die gleiche ist, mit der die Schnecken- $\nmid \nmid$ am natürlichen Standort ihren Laich ablegen.

\section{a) Geschlechtlichkeit bei Lunatia nitida}

Bei den Aquariumsversuchen erwies sich als sehr nachteilig, daß über die Unterscheidung der Geschlechter bei lebenden Lunatia, die sich in das Gehäuse zurückgezogen haben, nach äußeren Schalenmerkmalen nichts bekannt ist. Obgleich die meisten Vorderkiemerschnecken getrenntgeschlechtlich sind, liegen nur sehr wenige Angaben über die Ausbildung von sekundären Geschlechtsmerkmalen vor, die, am Gehäuse erkennbar, eine Trennung von $O^{\text {th }}$ und $q$ ermöglichen.

Nach einigen Autoren bestehen Unterschiede meist in der Schalengröße: Die Männchen sind kleiner (z. B. bei Assiminea nach van Benthem-Jutting), und in extremen Fällen kann fast von "Zwergmännchen" gesprochen werden (Lacuna pallidula, aus ANkel 1936). Nach THOrson ${ }^{3}$ ) ergibt sich bei den mei-

3) Herrn Prof, Dr. Gunnar Thorson, Helsingor, für die briefliche Mitteilung und für andere wertvolle Literaturhinweise auch an dieser Stelle herzlichen Dank. 


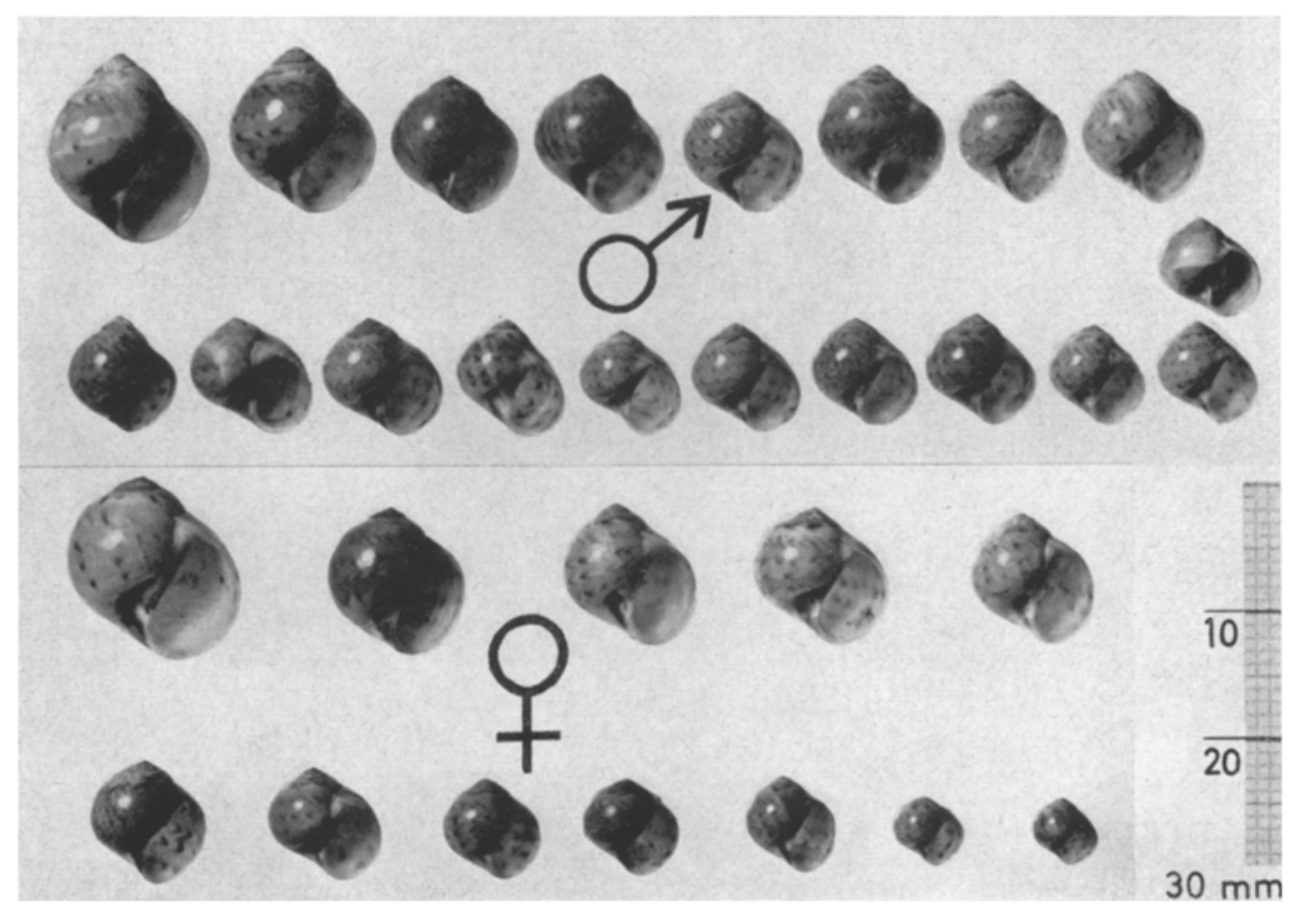

Abb. 6. Zusammenstellung von L. nitida-Schalen, bei denen die Zugehörigkeit der Geschlechter nach Entfernen des Weichkörpers und Präparation eindeutig ermittelt wurde.

sten Prosobranchiern als einzige Möglichkeit, die Geschlechter nach äußeren Schalenmerkmalen zu trennen, die Verschiedenheit der Gehäusegrößen. Die $\sigma^{7} \sigma^{7}$ sind "beinahe immer kleiner" und haben eine kürzere Lebensdauer als die 9 ㅇ.

Von anderen Schalenmerkmalen, die eine Unterscheidung der Geschlechter gestatten, erwähnt Ankel 1936, daß bei Viviparus viviparus, Hydrobia stagnalis und Margarites helicinus, "die Windungen der Schale beim $q$ etwas mehr aufgetrieben, stärker gewölbt oder plumper, die Nahtlinien weniger tief eingesenkt" sind. Ob diese angegebene subjektive Charakterisierung eine eindeutige Trennung der beiden Geschlechter ohne exaktes Schalenvergleichsmaterial gestattet, bleibt auch wegen der bei vielen Gastropoden-Arten ausgeprägten Variationsfähigkeit im Schalenbau sehr fraglich.

Nach Untersuchungen zahlreicher L. nitida, die aus Dretsch- und Bodengreiferproben in fixiertem oder betäubtem Zustand entschalt wurden, wobei das Gehäuse erhalten blieb, zeigten sich nach der Präparation des Weichkörpers an den entsprechenden Schalen keine Unterschiede, die auf einen Geschlechtsdimorphismus hindeuten könnten. Eine Gegenüberstellung von $\sigma^{\prime-}$ und $q$ Schalen (Abb. 6) etwa im Zahlenverhältnis der Geschlechter, bei denen, nach den hier durchgeführten Auszählungen, die Männchen mit rund $60 \%$ überwiegen, demonstriert die Schwierigkeit, nach äußeren Schalenmerkmalen (Größe, Form und Dicke der Schale, Lage und Ausdehnung der Mündung, Anzahl der Windungen und Form des Apex sowie der Nähte) bei eingezogenem Schneckenkörper die Geschlechter festzustellen. Beim Heraussuchen von $L$. nitida für den Besatz der Aquarien diente mitunter als Hilfsmittel die Ver- 


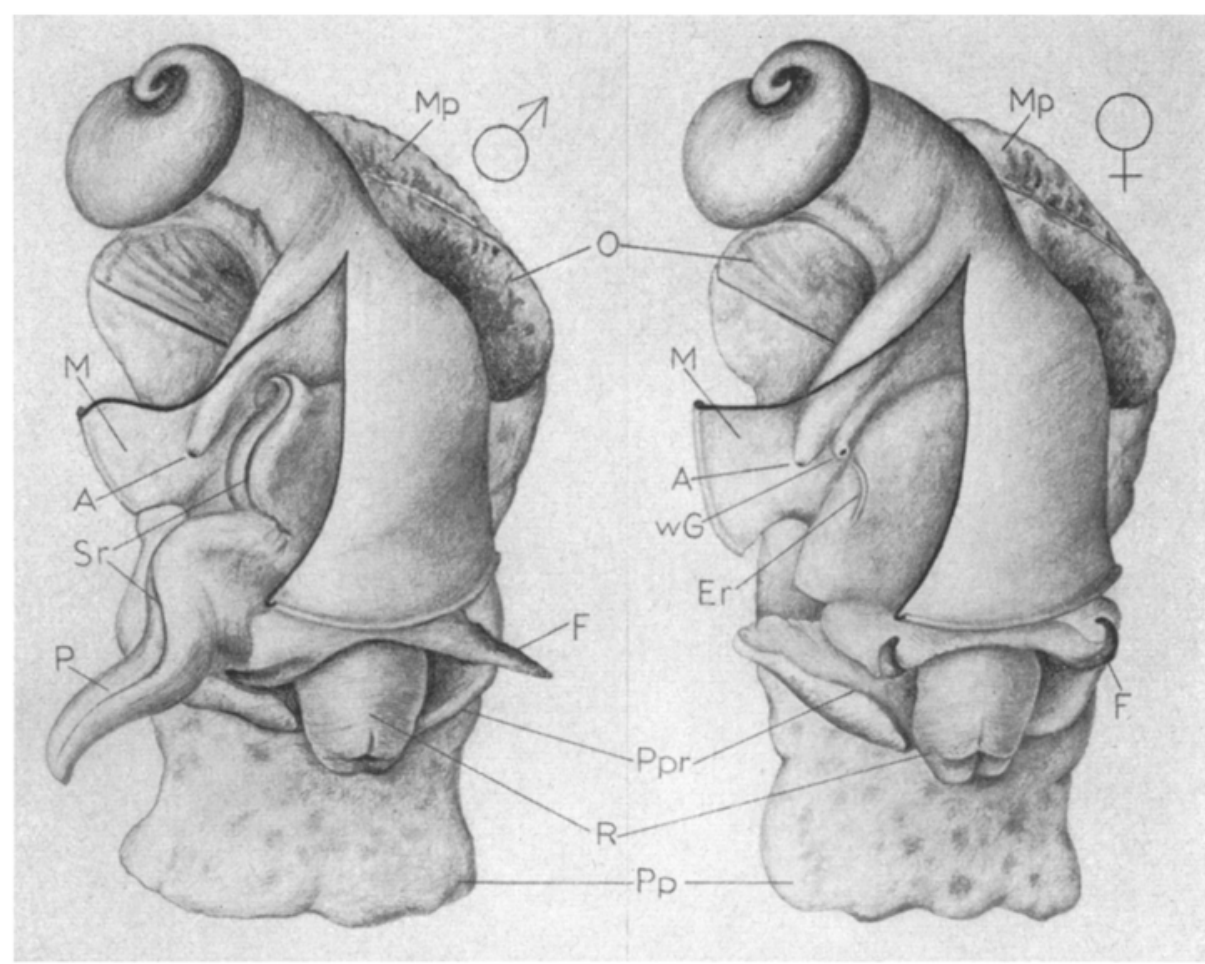

Abb. 7. Lunatia nitida- $\hat{\delta}$ und - + , ohne Schale. Der Mantelraum mit einem Schnitt geöffnet, rechter Mantelteil umgeschlagen. In beiden Zeichnungen ist nur das für die Fragestellungen der vorliegenden Darstellung Wesentliche ausgeführt worden. $A=$ After, $\mathrm{Er}=$ Eirinne, $\mathrm{F}=$ Fühler, $\mathrm{M}=$ Mantel, $\mathrm{Mp}=$ Metapodium (hinterer Fußabschnitt), $\mathrm{O}=$ Operculum, $\mathrm{P}=$ Penis, $\mathrm{P}_{\mathrm{p}}=$ Propodium (vorderer Fußabschnitt), $\mathrm{R}=$ Rüssel, $\mathrm{Sr}=$ Samenrinne, $w \mathrm{G}=$ weiblicher Geschledhtsporus. Vergr. $4 \times$.

schiedenheit der Nabelform. Bei größeren Tieren (mehr als $10 \mathrm{~mm}$ Gehäusehöhe) konnte beobachtet werden, daß der Nabel bei den $q$ q häufiger schmaler und in Richtung auf die Nahtecke spitzer ist als bei den $0^{7} \sigma^{7}$. Besitzt eine sehr große Schnecke einen schmalen, im Querschnitt zugespitzten und leicht gebogenen Nabel, dann handelt es sich fast immer um ein Q. Da aber $L$. nitida auch in der Ausbildung des Nabels \pm stark variiert, ist die Anwendung der Nabelform als Merkmal für die Geschlechtsbestimmung ebenfals nur sehr bedingt brauchbar. Eine eindeutige Geschlechtertrennung läßt sich deshalb nur nach der Präparation durchführen.

Es würde über den Rahmen der vorliegenden Darstellung hinausgehen, die Morphologie und Histologie der Geschlechtsapparate von L. nitida eingehend zu behandeln. Im Zusammenhang mit der Herstellung der Laichringe können daher nur die äußeren Genitalorgane, insbesondere die des Weibchens, besprochen werden, soweit dies für das Verständnis des Laichaktes, der Form des Geleges und der Verteilung der Eier im Laichring notwendig ist.

Das Männchen (Abb. 7) besitzt einen gut entwickelten Penis, der in der Ruhe, nach hinten umgeschlagen, stets unter dem Mantel liegt. Die deutlich erkennbare Samenrinne entspringt auf der rechten Seite des Kopffußes innerhalb der Mantelhöhle in der Nähe des Afters und läßt sich bis zur Spitze der Rute verfolgen. Der bei lebenden Tieren schwach rötlich gefärbte Penis ist 
pfriemenförmig, im Querschnitt nur wenig abgeflacht und besitzt keine Anhangsgebilde, wie sie z. B. bei Littorina stark ausgeprägt sind. Linke nennt diese Penispapillen bei den Littorinen „Klebdrüsen“, denen als Aufgabe die „Fixierung des männlichen Begattungsorganes am weiblichen Körper“ zukommt.

Verständlicher wird die Funktion der Drüsenanhänge am Penis beim Vergleich der Geschlechtsapparate von Tieren aus verschiedenen Biotopen. Die Littorinen leben in der Epifauna, L. littorea z. B. in einem Bezirk, der durch Brandung und Wellenschlag mitunter stark beeinflußt wird. Hier sind $\mathrm{Be}-$ festigungsmittel während der Begattung notwendig, auch wenn, wie LinkE berichtet, die Kopulation nur während des Niedrigwassers stattfindet. Penisanhänge sind nach ANKEL (1936) auch bei den beiden epibiotisch lebenden Schnecken Assiminea grayana (mit Drüsenpapillen) und Bittynia tentaculata (mit einem Drüsenschlauch) festgestellt worden. Die Naticiden, zur Endobiose gehörend, brauchen für den Begattungsakt in dem meist ruhigen Sediment keine Halteeinrichtungen.

Eine Kopulation konnte bei $L$. nitida bisher noch nicht beobachtet werden. Nach einer am 11. 4. 59 im Aquarium außerhalb des Sediments erfolgten Begattung eines L. catenaPärchens wurde die Länge des erigierten Penis (Gehäusehöhe des $\hat{\delta}=23 \mathrm{~mm}$ ) auf etwa $25 \mathrm{~mm}$ geschätzt. Das Männchen saß, etwas nach rechts verkantet, auf dem Gehäuse des Weibchens. Die Dauer des Begattungsaktes betrug etwa 5 Minuten. Diese Feststellung, die sich jedoch nur auf eine einmalige Beobachtung stützt, deckt sich nicht mit den Ergebnissen von Bouchard-Chantereaux, der nach Jefrreys 1867 mitteilt, daß bei $L$. catena "the sexual coition lasts many hours". Die einmalige Beobachtung — obgleich die Aquarien vor- und nachher unter ständiger Kontrolle standen - läßt nicht den Schluß zu, daß während der Brunstzeit nur eine Kopula stattfindet. Leider starben die Schnecken im Sommer 1959, das catena- 9 legte kein Laichband $a b$.

Offen und weiteren Untersuchungen vorbehalten bleiben zunächst auch folgende Fragen, bei deren Lösungen die eingehende Morphologic und Histologie der männlichen und weiblichen Genitalorgane von $L$, nitida besonders zu berücksichtigen sind. 1. Welche Zeit vergeht von der Kopulation bis zur Laichabgabe? Bei L. nitida sheint das Laichen nicht unmittelbar nad der Begattung zu erfolgen, wie Linke dies für Littorina littorea mitteilt, bei der "schon wenige Stunden nach der Kopula" die Laichabgabe beginnt. Von den im späten Herbst eingebrachten lebenden $L$. nitid $\left.a^{4}\right)$ laichten die Tiere im nächsten Frühjahr, nachdem die Schnekken monatelang einzeln in den Aquarien gehalten wurden. 2. Wann wird L. nitida geschlechtsreif und wie viele Laichperioden können bei cinem Weibchen ermittelt werden?

Die weibliche Geschlechtsöffnung ist bei lebend-frischen L. nitida, die nach Betäubung entschalt und präpariert wurden, sehr schwer zu erkennen. Erst bei fixierten Schnecken hebt sich der Endabschnitt des Uterus - nach Ankel (1936) und Haller (1893) die "Vagina" - etwas deutlicher, in der Nähe des Afters liegend, aus dem Mantel heraus (Abb. 7). Genau unter dem Genitalporus beginnt am Kopffuß eine Rinne, die in einer leichten Krümmung in Richtung auf die Basis des rechten Fühlers verläuft und etwa nach dem ersten Drittel zwischen der Geschlechtsöffnung und dem rechten Tentakel mit einem kurzem Abflachen aufhört. Die Rinne wird von einer Faltenduplikatur des Kopffußepithels gebildet und fällt bei fixierten Tieren wegen der hellen gelblichen Tönung sofort auf. Diese Bildung des weiblichen Genitalapparates bei $L$. nitida entspricht der von Linke bei Littorina beschriebenen "Aufwulstung, die sich vom Porus des Uterus nach vorn bis unter den rechten Tentakel

4) Für den unermüdlichen und umsichtigen Einsatz bei der Beschaffung von lebenden Lunatia nitida sei auch an dieser Stelle dem Kapitän des Forschungskutters "Uthörn“ der Biologischen Anstalt Helgoland, Herrn T. Hornsmann, sowie der gesamten Schiffsbesatzung herzlichst gedankt. 
an der rechten Seite des Kopffußes hinzieht und wahrscheinlich eine Art Ovipositor darstellt". Haller berichtet über die "Vagina" bei Strombus gigas: „Diese mündet in eine lange, auf der rechten Seite des Vorfußes (Propodium) bis zu dessen Ende hinziehende Rinne. Nach oben zu wird diese Eileiterrinne von einer mächtigen Hautfalte überdeckt." Die Bezeichnung "Eileiterrinne“ kann zu Mißverständnissen führen, und die von LiNkE gewählte Benennung "Ovipositor" entspricht nicht dem eigentlichen Sinn dieses Begriffes. Eine gewisse Berechtigung für die Anwendung der letzteren Bezeichnung bei Gastropoden läge vor nach Beobachtungen von Sімкотн - er fügt einschränkend hinzu - an "eingetrocknetem Materiale" von Janthina exigua, bei der "die Scheide als ein langes Rohr im Nacken hervorgestreckt werden und als Ovipositor dienen würde, um die Eicocons an dem Floß zu befestigen".

Über die Funktion dieser mit "Ovipositor" bezeichneten "Furchen" am Kopffuß bei Litlorina sagt LINKE, daß sie wegen ihres „einheitlichen" Schleimdrüsenepithels wohl „die derbe, äußerste Membran des Laiches von L. litto-

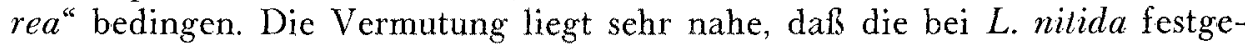
stellte Rinne weiterhin den Zweck hat, die während des Laichaktes aus dem Uterus tretenden Eier in eine bestimmte Richtung zu leiten. Es wird daher vorgeschlagen, diese Bildung mit „Eirinne" zu benennen. Obgleich vorerst nur wenig Untersuchungsmaterial von anderen Naticiden vorliegt, deutet aber der Vergleich der Eirinnen bei L. nitida und Polynices josephinus bereits darauf hin, daß Zusammenhänge bestehen zwischen der Form und dem Verlauf der Rinne und der Verteilung sowie Anordnung der Eier im Laichgelege, worauf bei der Darstellung des Laichvorganges noch kurz eingegangen wird.

\section{b) Fruchtbarkeit der nitida- 99 und Laichzeiten}

Bei 3 Weibchen ist die Laichablage im Aquarium kontinuierlich beobachtet worden: 19 Laichringe vom 9. 9.57-8. 2. 58 (mit einer längeren Unterbrechung vom 9. 9.-1. Laichring bis 14.11.57-2. Laichring), 13 Laichringe vom 31. 3.-24. 6. 59, 11 Laichringe vom 30. 3.-31. 5. 60.

Nach einer Tabelle aus Ankel (1936) laicht L. nitida im Juni. Thorson fand im Kattegat im Juli Laichringe. Auf Grund der Beobachtungen über Laichgelege, die bei den Aquariumsversuchen mit Lunatia seit 1956 nebenher registriert werden konnten, ist sehr wahrscheinlich, daß die Nabelschnecken während des ganzen Sommers laichen.

Als Intervall zwischen den einzelnen Laichringablagen wurden im Durchschnitt 4-5 Tage ermittelt. Obgleich die verhältnismäßig wenigen Beobachtungen noch nichts Endgültiges über die Gesamtzahl der von einem Weibchen abgelegten Laichringe aussagen, dürfen wir aber annehmen, daß die Laichperiode für L. nitida am natürlichen Standort mindestens 2 Monate dauert.

Nach der Zusammenstellung der Laichzeiten unter Aquariumsbedingungen (Tab. 3) könnten für die Fortpflanzungsperiode 4-6 Monate gerechnet werden. Auffallend in der Tabelle ist, daß in den beiden Jahren 1957/58 das Laichen im Aquarium nur in den Wintermonaten stattfand, während das $\mathrm{Ab}$ laichen 1959/60/61 im Februar bzw. März begann und mehr als ein halbes Jahr anhielt. Witterungsmäßig allein sind die Unterschiede nicht bedingt, sie sind vorwiegend mit der verschiedenen Zusammensetzung des eingebrachten lebenden Materials und dem Zeitpunkt des Überführens aus dem natürlichen Standort in die Aquarien zu erklären. Werden nitida-qㅇ während ihrer Laichperiode im Spätsommer gefangen, so wird, nach einer \pm langen Unter- 
Tabelle 3

Laichzeiten von Lunatia nitida im Aquarium

Januar
Februar
März
April
Mai
Juni
Juli
August
September
Oktober
November
Dezember

brechung, das Laichen im Aquarium fortgesetzt, die Laichperiode für diese Tiere wird verlängert (vg1. 1957/58). Bei später eingebrachten Tieren (Oktober, November) setzt der Laichbeginn im darauffolgenden Jahr früher ein, sehr wahrscheinlich wegen der günstigen Ernährungsbedingungen in den Aquarien, in denen die Schnecken den ganzen Winter über reichlich gefüttert wurden.

Über die auslösenden Faktoren des Laichbeginns bei L. nitida im Aquarium kann vorerst nur ausgesagt werden, daß die absolute Wassertemperatur nicht die entscheidende Rolle spielt, denn die $q$ begannen mit der Laichablage bei $5,3^{\circ} \mathrm{C}$ (min.) und max. bei $22,0^{\circ} \mathrm{C}$. Wichtiger scheint die Tendenz des Temperaturverlaufs zu sein.

\section{c) Direkte Beobachtung der Laichablage}

Die ersten Versuche zur Klärung der Technik, mit der L. nitida die Laichringe herstellt, wurden in Aquarien mit $5-8 \mathrm{~cm}$ Sedimenthöhe durchgeführt. Die Korngrößen des Bodenmaterials wurden verschíeden gewählt, von etwas schlickigem feinem bis zu kiesigem groben Sand. Die Aquariumsanlage bestand entweder aus einzeln belüfteten Becken oder aus einer Serie von Aquarien, die mit Filter zu einem kleinen geschlossenen Kreislauf zusammengestellt waren. Die Aufstellung der Aquarien erfolgte in verschieden-, jedoch nicht konstant-temperierten Räumen, die Wassertemperaturen wurden ständig kontrolliert. Als Futter erhielten die Schnecken lebende Muscheln (vor allem Angulus fabula und Spisula solida sowie S. subtruncata), deren Schalen sie durchbohrten, um dann den Weichkörper auszufressen. Im Winter sind gelegentlich mittelgroße Mytilus edulis aufgeschnitten in die Becken gelegt worden, wo die Weichkörperhälften in den Klappen von den Schnecken, die hierbei nicht zu bohren brauchten, sofort angenommen und in verhältnismäßig kurzer Zeit aufgezehrt wurden.

Bei den Aquariumsversuchen zur Ermittlung der Laichtechnik ergaben sich anfänglich Schwierigkeiten vor allem in der Feststellung des Laichaktbeginns, weil 1) eine Vorbereitungsphase bei L. nitida fehlt, die dem Laich- 
akt vorangeht, wie sie Gigliol als „incipient stage“ bei Polynices triseriata und $P$. heros beobachten konnte. Diese Schnecken liegen vor Laichbeginn während der Ebbe außerhalb des Wattbodens auf der Seite. Schleimabsonderungen aus dem vorderen Teil der Kriechsohle, die durch Umschlagen der Ränder des Pro- und Metapodium nach unten eine muldenartige Höhlung bildet, formen sich im auflaufenden Wasser zu langen Girlanden und leiten so den Laichakt ein. Den ausgeschiedenen Schleim bezeichnet Gigırou als ein "precursor of the matrix collar jelly". 2) Das Laichen der nitida- $Q \oint$ findet zu verschiedenen Tageszeiten statt. Etwa \%/3 aller aus den Aquarien entnommenen Laichringe wurden während der Nacht abgelegt. 3) Die Tiefe, in der die laichenden Schnecken im Sediment sitzen, kann sehr verschieden sein. Bei einer ca. 2$3 \mathrm{~cm}$ im Sand ablaichenden Lunatia ist auf der Sedimentoberfläche nichts zu erkennen.

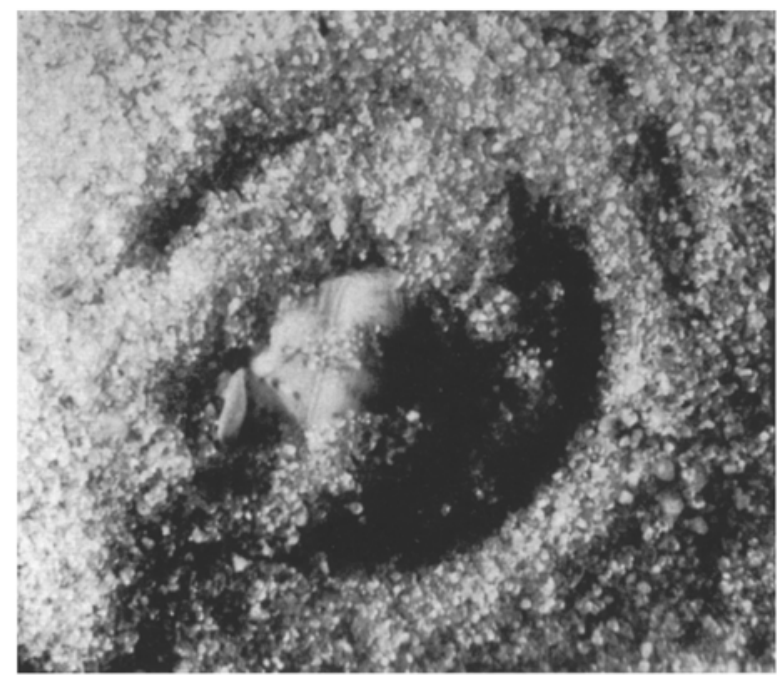

Abb. 8. Ringwall, der auf dem Sand zu erkennen ist, wenn ein $L$. nitida- 9 dicht unter der Sedimentoberfläche ihren Laichring formt. Vergr. $1^{1 / 2} \times$.

Durch Zufall kann jedoch die Beobachtung zustande kommen, wenn ein nitida-O den Laichring dicht unter der Oberfläche des Sediments herstellt. In diesen Fällen deutet ein Ringwall auf dem Sand den Laichvorgang an, zuweilen ist vom laichenden $O$ das Gehäuse und der hochgeschlagene linke Vorderrand des Propodium zu erkennen (Abb. 8). Obgleich bei den Schnecken in dieser Stellung vom eigentlichen Laichvorgang, vor allem von der Herstellung des Ringes selbst, nur wenig zu erkennen ist, lassen sich jedoch 2 Vorgänge beobachten, die für den Laichakt wesentlich sind: 1) Das Weibchen sitzt normal orientiert im Sediment, d. h. das Gehäuse etwa in der Lage wie beim Kriechen (vgl. Abb. 11). Deutlich ist zu sehen, daß eine schubweise Drehung des Tieres um seine Schale als Achse im entgegengesetzten Uhrzeigersinne erfolgt. Der langsam ausgeführte Schub mit der schwachen Drehung des Gehäuses ist vor allem an der Bewegung des hochgeschlagenen Teiles des Propodium zu erkennen, das beim Drehen wie eine Pflugschar im Sediment wirkt. Die Zeit zwischen den einzelnen Schüben, in der sich das $q$ bei dieser Beobachtungsmöglichkeit scheinbar in völliger Ruhe befindet, beträgt nach zahlreichen Messungen in mittelkörnigem Sand im Durchschnitt $67 \mathrm{sec} .(\max .=105, \mathrm{~min} .=45 \mathrm{sec}$.$) .$ 
2) Nach Fertigstellung des Laichringes gräbt sich die Schnecke etwas tiefer in den Sandboden und kriecht, wieder im entgegengesetzten Uhrzeigersinn, so unter dem Außenrand des noch vom Sand bedeckten Ringes kreisförmig herum, daß sie mit ihrem Gehäuse den Laichring auf die Sedimentoberfläche hebt. Dieses "Trimmen" ist die Schlußphase des Laichaktes. Sobald der gesamte Ring auf dem Sand liegt, verläßt das Weibchen die Stelle, kriecht zuerst auf dem Sediment davon, um sich aber bald wieder einzugraben.

Das Trimmen gehört zum Handlungskomplex des Laichaktes und wird von jedem nitida- 9 durchgeführt. Nur in 2 Fällen konnten während der

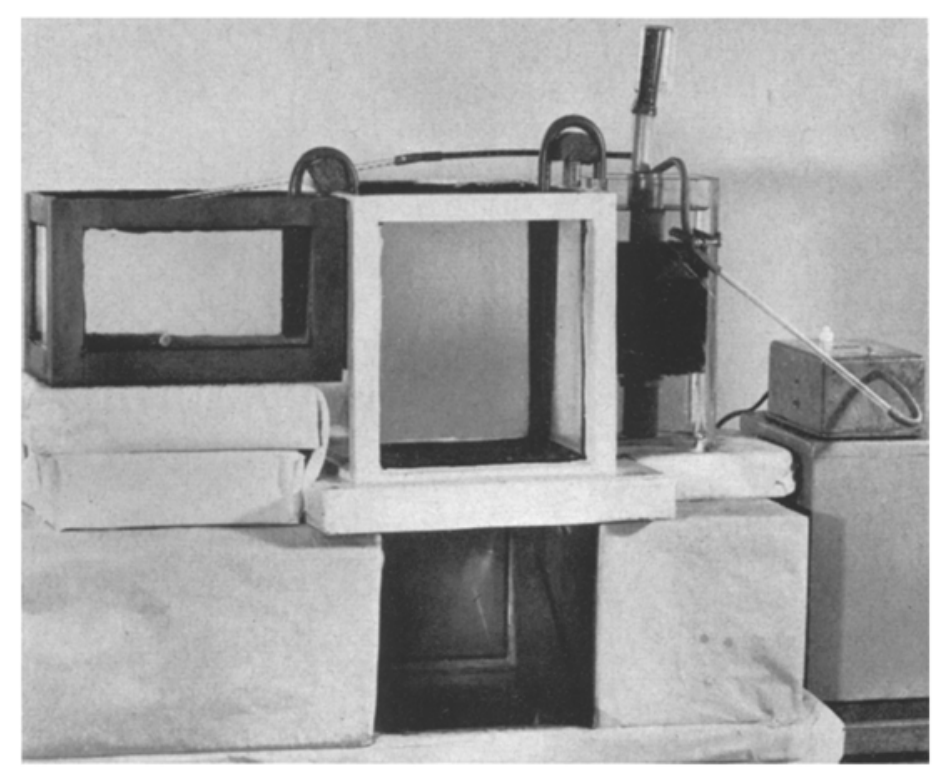

Abb. 9. Aquariumseinrichtung für die Beobachtung des Beckenbodens von unten mit Hilfe eines um ca. $45^{\circ}$ geneigten Spiegels. Ein kleiner geschlossener Kreislauf mit Filter únd 2 Bekken. Das linke Aquarium dient zur Hälterung, unter dem mittleren befindet sich der Planspiegel. Maße des weißen Versuchsbeckens: Grundfläche $22 \times 92 \mathrm{~cm}$, Höhe (ohne Grundplatte) $=25 \mathrm{~cm}$

Aquariumsversuche je ein Laichring aus dem Sediment gewühlt werden, die von den betreffenden Schnecken nicht gehoben waren. Die sich entwickelnden Eier waren ca. 3 Tage alt. Sehr wahrscheinlich sind die beiden Tiere durch irgendeine, nicht bekannte Störung am Trimmen gehindert worden.

Bei der eben geschilderten, bisher angewandten Methode, die nur eine zufällige und unvollständige Beobachtung der Laichablage bei L. nitida ermöglichte, blieben ungelöst: a) Wie wird der Ring vom $q$ gebildet, b) wodurch erhält er seine typische Form, c) wie gelangen die Eier in das Gelege und schießlich d) welche Technik bedingt die etwa gleiche Korngröße des im Laichring eingebauten Sediments?

Zur Beantwortung dieser Fragen und zur direkten Beobachtung der Laichringablage verhalf ein Verfahren, das sich bereits bei früheren Versuchen gut bewährt hatte (Zregelmerer), zum Erfolg.

Ein Aquarium (Grundfläche $=22 \times 22 \mathrm{~cm}$, Höhe $=25 \mathrm{~cm}$ ) wurde so aufgestellt, daß ein um etwa $45^{\circ}$ geneigter Spiegel unter dem Becken eine gleichzeitige Beobachtung der Tiere von oben und unten gestattete (Abb. 9). 
Die Sandschicht im Aquarium betrug max. $10 \mathrm{~mm}$. Um die laichenden Weibchen ist während des Laichaktes mit einer Pipette soviel Sediment entfernt worden, daß den Schnecken für die Laichringherstellung noch genügend Baumaterial zur Verfügung stand, und die Voraussetzungen für die direkte Beobachtung gegeben waren.
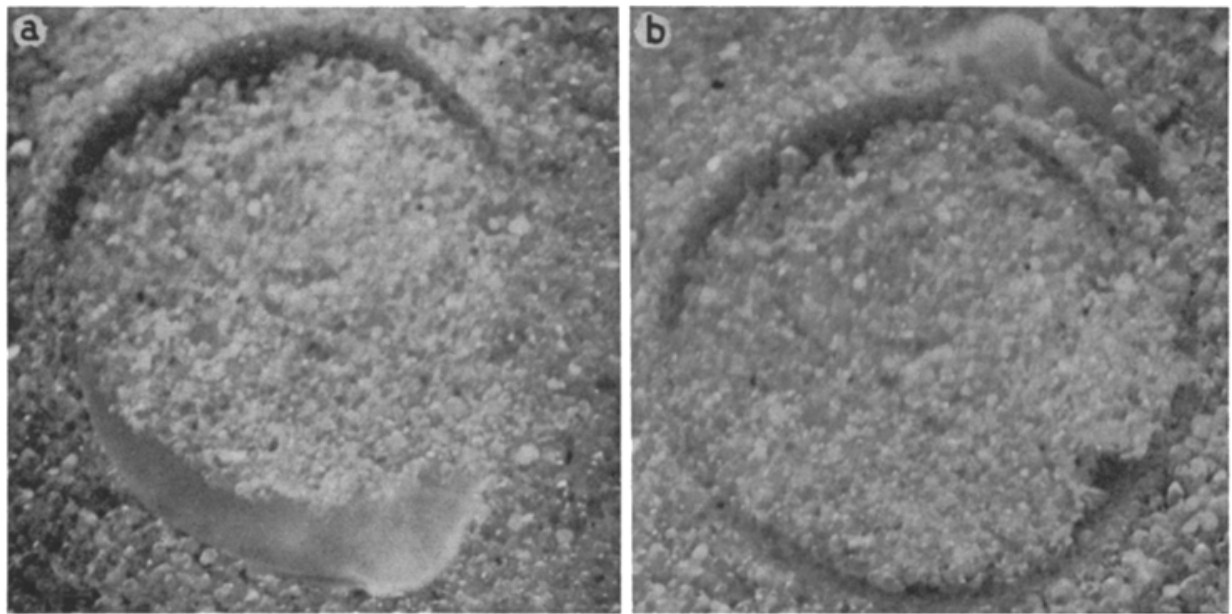

Abb. 10. Photographische Aufnahmen durch den Planspiegel während der Laichringherstellung von $L$. nitida. Die seitenverkehrten Bilder geben die richtige Orientierung des $O$ beim Laichakt von oben gesehen. a $=$ mehr als $1 / 2$ Windung des Ringes ist fertiggestellt, der am oberen dunkleren Kreisbogen zu erkennen ist, darunter der von der Schnecke nach rechts weit ausgedehnte Fuß mit dem rechten Propodiumteil [a) entspricht etwa der Stellung 2 in Abb. 12]. $b=$ Dasselbe $O$ hat mit dem Propodium den Ringanfang erreicht und, mit ihrer Kriechsohle auf der 1. Windung entlanggleitend, dreht sich L. nitida schubweise im entgegengesetzten Uhrzeigersinne weiter [b) entspricht fast der Stellung 3 in Abb. 12]. Vergr. ${ }^{3 / 3} Y_{.}$.

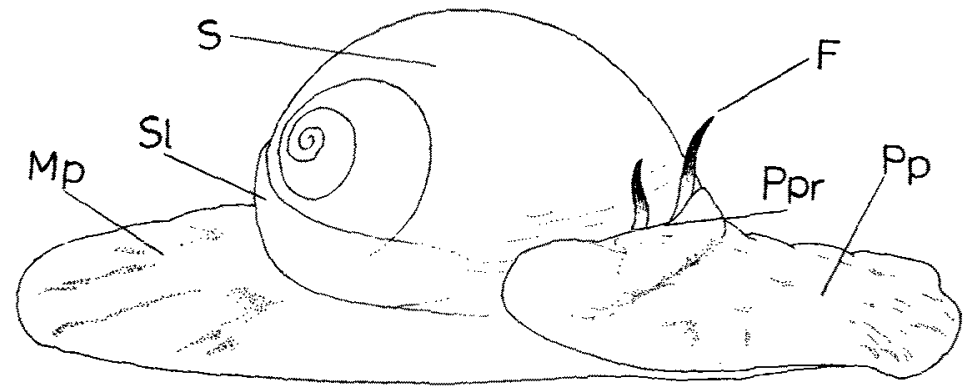

Abb. 11. Lumatia nitida auf der Sedimentoberfläche kriechend, von der rechten Seite gesehen Das Operculum wird beim Kriechen vom Schalenlappen des Metapodium verdeckt. $\mathrm{F} \approx$ Fühler, $\mathrm{Mp}=$ Metapodium, $\mathrm{Pp}=$ Propodium, $\mathrm{Ppr}=$ hinterer oberer Rand des Propodium; punktiert angedeutet die beim Laichakt wellenförmig von rechts nach links laufende Falte (Siebebewegungen) während des Einbringens von Sandkörnchen in die darunter liegende Höhlung. $\mathrm{S}=$ Schale, $\mathrm{Sl}=$ über einen Teil der Schale gezogener Lappen des Metapodium. Dieser Lappen kann vom Tier fast bis über das ganze Gehäuse gezogen werden. Vergr. $4 \times$.

Die ständige Kontrolle des Verhaltens der Tiere in den Becken ließ den Laichbeginn im Spiegel an der typischen Stellung der nitida-우 eindeutig erkennen (Abb. 10). Die rechte Seite des Pro- und Metapodium ist etwa zu einem Halbkreissektor stark ausgedehnt (Abb. 12, Stellung 1), die linke ent- 
sprechend eingezogen. Mit der Ausdehnung des Propodium, die zum Teil von der Größe des Tieres abhängig ist, außerdem von der vom Weibchen gesteuerten Intensität der Schwellung, sind der äußere $\phi$ und die Bandbreite des Laichringes gegeben.

Die Haupttätigkeit beim Laichringbau führt das Propodium aus. Der bei den Naticiden gut entwickelte Fußabschnitt (Abb. 11) bildet nach Simrotr "ein nach oben und hinten auf Kopf und Schale hinaufgeschlagenes, beim Graben schützendes Schild" und befähigt die Vertreter dieser Familie zu den Lokomotionsleistungen in den verschiedenen Sedimenten. Gigloul bezeichnet diesen Abschnitt mit ,frontal apron or sub-tentacular fold“. Unter dem "Schild" befindet sich vor dem Kopffuß und dem überdeckten vorderen Schalenrand eine Höhlung, deren Epithel Schleim absondert. Während des Laichaktes (Abb. 12) bewegt sich ständig eine auf den oberen Propodiumrand beschränkte Falte wellenförmig von rechts nach links in der Zeit von etwa 1 sec. Die spitzwinklig-dreieckige Randfalte ist bei Beginn schmaler, im Bereich der Mitte zwischen den beiden Fühlern am breitesten und gegen die linke Seite hin wieder kleiner. Durch den von rechts nach links wandernden Spalt fallen nur die Sandkörnchen, deren Korngröße geringer ist als die Spaltbreite, in die vorher beschriebene Höhlung. Mit Hilfe dieser Siebetätigkeit wird die Korngrößenauswahl des im Laichring eingebauten Sedimentanteils ermöglicht. In der Höhlung wird das so eingebrachte Material mit Schleim vermischt. Nachdem die Höhle gefüllt ist, setzen für eine kurze Zeit die Siebebewegungen des Propodiumrandes aus, und das $q$ dreht sich mit einem kurzen, langsam ausgeführten Schub im entgegengesetzten Uhrzeigersinne weiter. Dabei drückt die Schnecke das Sand-Schleim-Gemisch so aus der Höhlung unter der rechten Kante des Propodium-Schildes, daß es plan auf den nach rechts ausgedehnten Fußteil zu liegen kommt und nach dem Hartwerden des Schleimes im Seewasser die charakteristische Form erhält.

Während die eben geschilderten Tätigkeiten beim Laichakt einer Lunatia gut zu verfolgen sind, entzieht sich der Einbau der Eier in das Laichringsubstrat der direkten Beobachtung. Nach der Lage der äußeren weiblichen Geschlechtsorgane und der Form der Eirinne läßt sich nach Kenntnis der sonstigen Tätigkeiten einer L. nitida beim Laichen über den Weg der aus dem Uterus austretenden Eier bis in den Laichring folgendes aussagen: Im Augenblick, in dem das Tier die Sand-Schleim-Füllung herausdrückt, tritt eine Anzahl von Eiern aus dem Uterus. Sie werden zuerst gerichtet auf der Eirinne befördert - hierbei erhalten die Eier wahrscheinlich ihre Hülle - um dann ungeordnet über den Boden des Kopffußes in die noch Schleim-klebrige Laichringmasse zu gelangen, bevor diese auf der rechten Fußseite ausgebreitet, endgültig geformt wird. So ist einmal zu erklären, daß wir im nitida-Laichring und auf der Außen- sowie Innenfläche die festgehefteten Eier finden, zum anderen, daß, wegen der portionsweisen Abgabe von Eiern aus dem Uterus, die zuerst in eine bestimmte Richtung geleitet, dann regellos auf das Baumaterial stoßen, sich gelegentlich Anhäufungen von Eiern an verschiedenen Stellen im Laichring bilden.

Bei Polynices josephinus liegen die Eier regelmäßig im Laichband, jedes $\mathrm{E}$ in einer hohlkugelförmigen Höhle mit fast konstantem Abstand in einer Ebene im verkitteten Sediment eingebettet. Die Eier benötigen daher nach dem Verlassen des Uterus eine gerichtete Zuführung von der weiblichen Genitalöffnung bis zu der Stelle, an der sie in die Laichbandmasse eingebaut werden. So ist beim $P$. josephinus- $q$ eine Eirinne zu erwarten, die nicht, wie bei $L$. nitida auf der Hälfte des Weges aufhört, sondern durchgehend bis an den Rand 

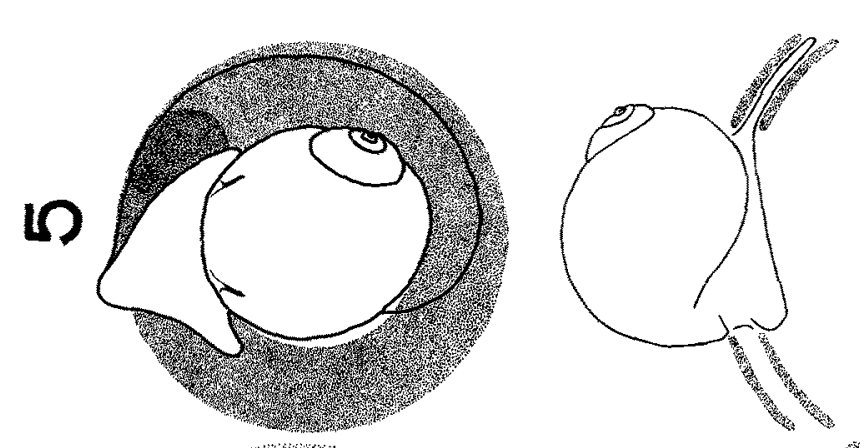

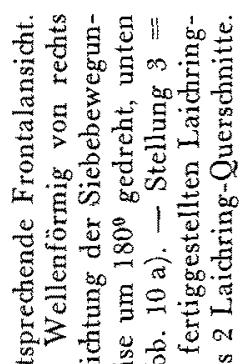

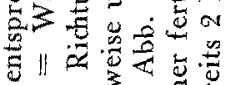
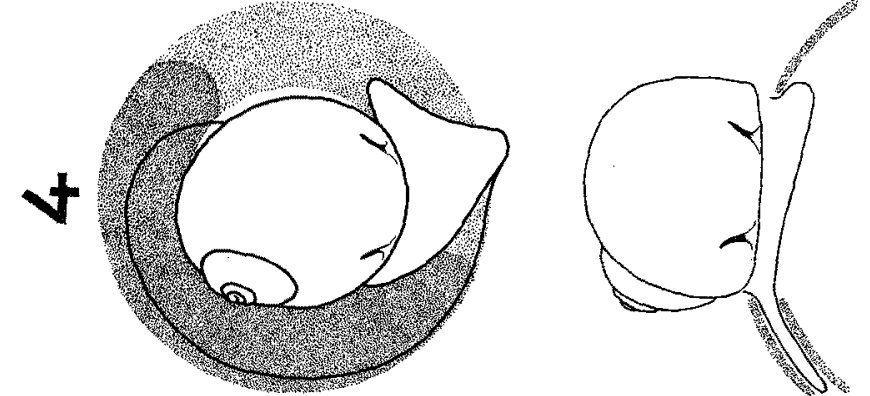

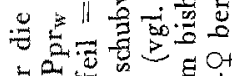

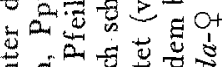

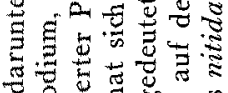

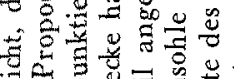

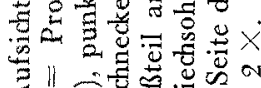

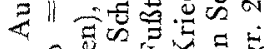

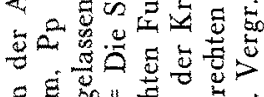
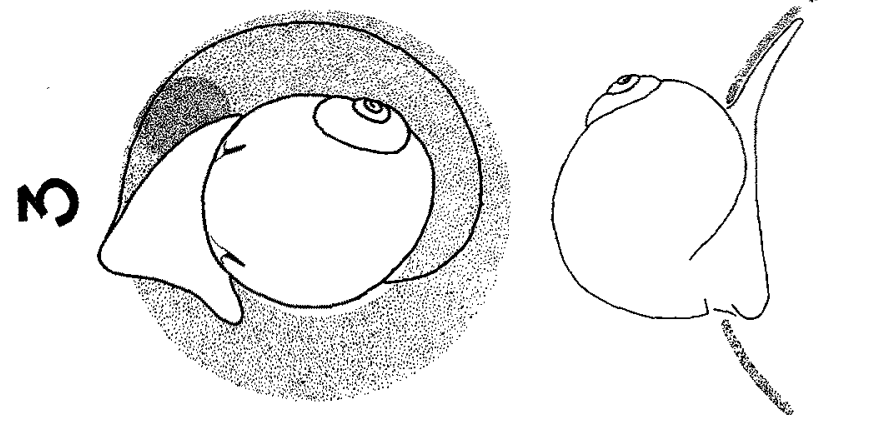

.

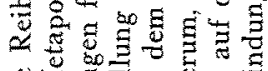

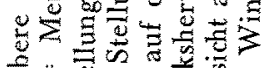
ธ||

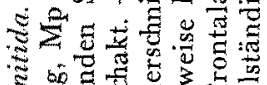

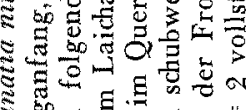

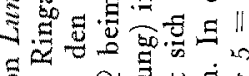

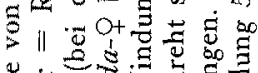
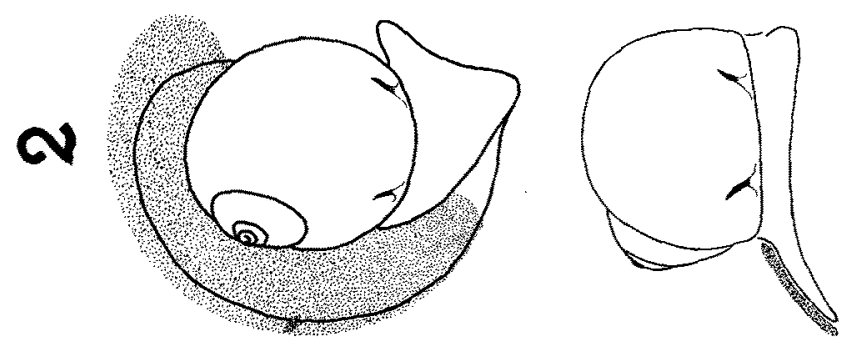

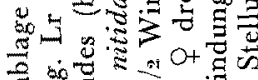

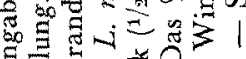

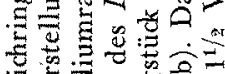

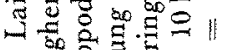

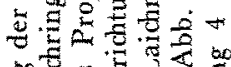

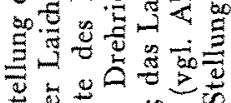
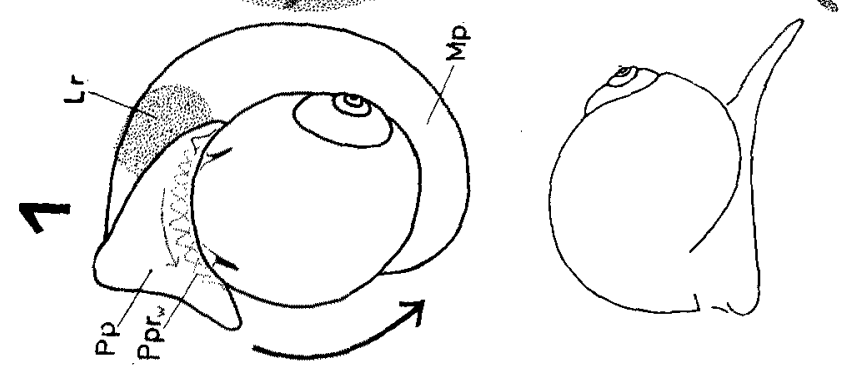

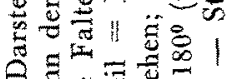
๑

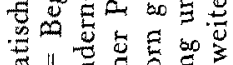

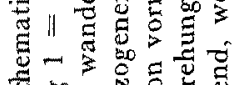

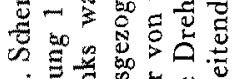

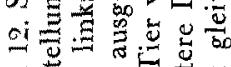

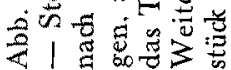


des Kopffußes in der Nähe der Basis des rechten Fühlers verläuft, was die Präparation von $P$. josephimus eindeutig bestätigt.

Nach dem Herausdrücken der Sand-Schleim-Masse, die den Laichring jedesmal um ein kleines Stück verlängert, setzt das nitida- $q$ die Siebebewegungen fort, die wieder bis zum nächsten Schub anhalten. Die sich auf diese Art schubweise links drehende Schnecke benötigt für eine Windung (nach Beobachtungen an laichenden Tieren im Aquarium) 11/2-21/2 Stunden. Verständlicherweise dauert die Herstellung eines Ringes im gröberen bis groben und kiesigem Sediment länger, da häufigere Siebebewegungen notwendig sind, um die Höhlung mit Baumaterial in der entsprechenden Sandkorngröße zu füllen.

Wie die schematische Darstellung (Abb. 12) zeigt, dreht sich die Schnecke, nachdem sie eine Windung fertiggestellt hat, so weiter, daß der rechte Teil ihrer Kriechsohle auf dem bereits gelegten Ring (1. Windung) entlanggleitet. Hierbei wird auch die Übereinstimmung der Gehäusehöhe des Weibchens mit dem inneren $\phi$, dem eindeutigsten Maß des Laichringes, verständlich.

Bei Beginn der 3. Windung dreht sich das Tier in der beschriebenen Art schubweise mit dem rechten Teil der Kriechsohle auf der 2. Windung. Die Beendigung des Laichaktes ist am Aufhören der Siebebewegungen zu erkennen. Das nitida-Q verhält sich ca. 10 Minuten völlig ruhig, verschwindet dann plötzlich im Sand, um den Laichring, wie geschildert, auf die Sedimentoberfläche zu heben.

\section{Kurze Bemerkungen über die ersten Furchungsstadien und das Schlüpfen der Veligerlarven}

Bei der Durchsicht von frischen Laichringen mit mehr als 2 Windungen sind bei der Entnahme des Laiches aus dem Aquarium die zuerst abgelegten Eier am Ringanfang bereits einmal gefurcht. Beobachtungen an zahlreichen Laichringen ließen erkennen, daß die um etwa 1 Windung vorher abgelegten Eier um einen Furchungsschritt voraus sind. In Gelegen mit mehr als 3 Windungen liegen am Ringbeginn bereits 4-Zellenstadien, in der Mitte, d. h. in der gleichen Gegend der 2. Windung, 2-Zellenstadien, während die Eier am zuletzt hergestellten Ringteil noch keine Furche besitzen. In den unmittelbar nach dem Trimmen aus den Aquarien entnommenen Laichringen beginnen die ungefurchten Eier am Ringanfang nach 2-4 Stunden mit den Teilungen (Wassertemperatur um $18^{\circ} \mathrm{C}$ ). Die 3 Furchungen bis zum ersten Mikromerenquartett benötigen etwa $6-7$ Stunden, d. h. ungefähr alle 2 Stunden tritt eine Teilung ein, wie die Auswertung von Zeitrafferfilmaufnahmen zeigen (Abb. 13).

Der Filmstreifen dient neben der Möglichkeit, im Laufbild den Spiraltypus der total-inaequalen Furchung der Eier von Lunatia nitida zu demon-

Abb. 13. Die ersten 3 Furchungen des Eies von Lanatia nitida nach Zeitrafferfilmaufnahmen (Raffung $=4$ Bilder pro Minute, d. h. nach 14 Sekunden 1 Aufnahme mit 1 Sekunde Belich tung). Der Zeitabstand zwischen den einzelnen Stadien in der Abbildung beträgt 4 Minuten, jedes 16. Bild des Films wurde nachgezeichnet. Danach ergeben 15 Bilder in der Zeichnung den Entwidklungsablauf in 1 Stunde. Ungefurchtes Ei mit Hülle, die bei den folgenden Stadien fortgelassen wurde. Maßst. $=0,1 \mathrm{~mm}$. 


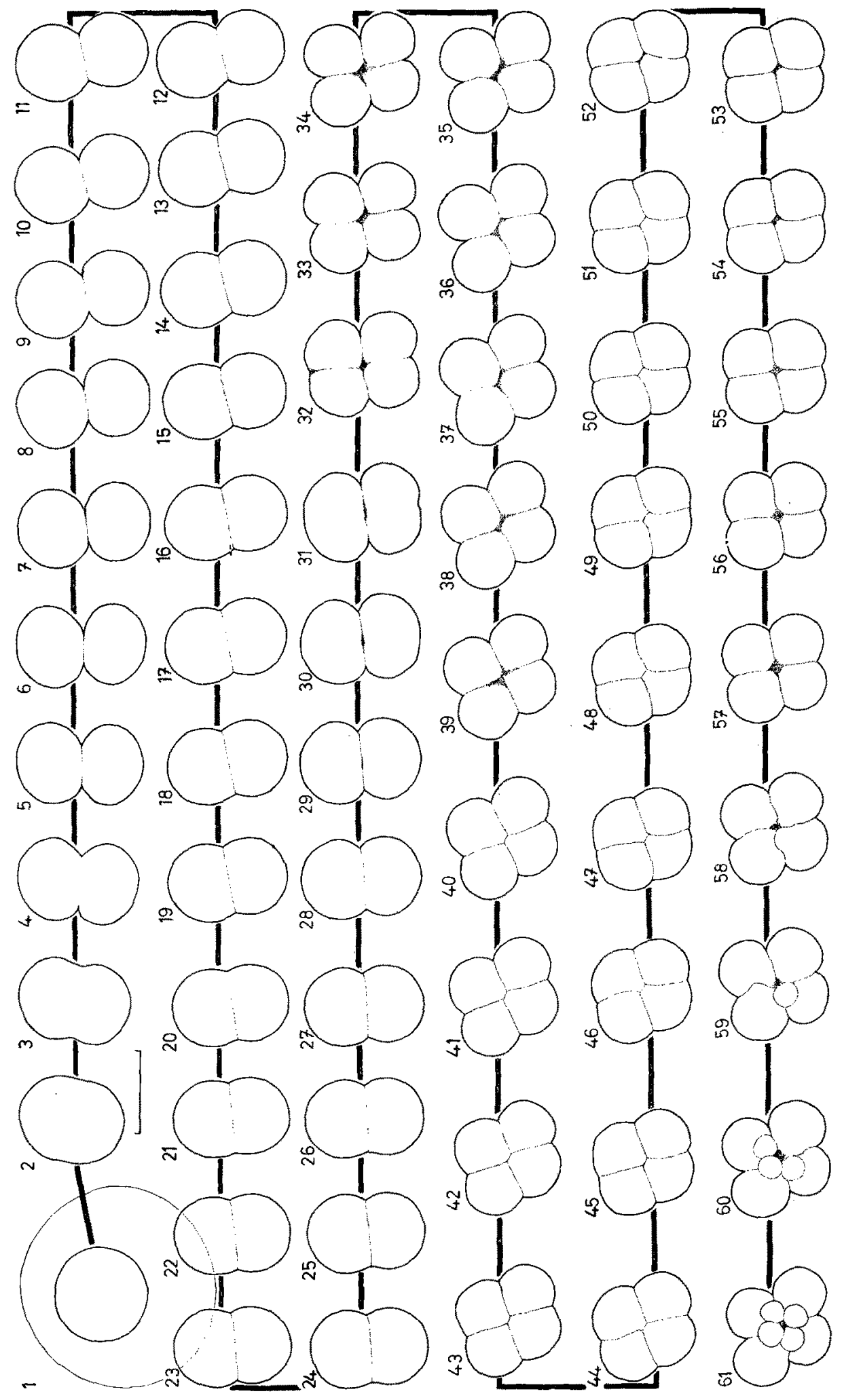


strieren, vor allem dem Zweck, mit Hilfe einer einfachen Auswertungsmethode die Formveränderungen der verschiedenen Stadien vom ungefurchten $\mathrm{Ei}$ bis zum ersten fertigen Mikromerenquartett darzustellen.

Beim 2- und 4-Zellenstadium liegen die Makromeren nach jedem Einschneiden der Furchen tief eingeschnürt aneinander, pressen sich dann stark zusammen, um vor der neuen Teilung wieder etwas auseinanderzuweichen.

Nach Hertling beträgt die Entwicklungsdauer vom abgelegten Ei bis zum Schlüpfen der Veligerlarven etwa 21 Tage bei einer mittleren Wassertemperatur von $8,48^{\circ} \mathrm{C}$ (zwischen $6,2-10,8^{\circ} \mathrm{C}$ ). Die Zeit in diesem Temperaturbereich konnte bestätigt werden. Bei $18-20^{\circ} \mathrm{C}$ schlüpften die Veliger nach 11-12 Tagen.

Um die bei der Entnahme den Laichringen anhaftenden, im Sediment lebenden und für die Eier schädlichen Ciliaten zu entfernen, ist folgende Methode angewandt worden: Unmittelbar nach dem Herausnehmen aus einem Becken wurde jeder Laichring für die Dauer von ca. $40-60$ sec. in Süßwasser mit der gleichen Temperatur gelegt, dann in gleichtemperiertes filtriertes Seewasser überführt. Am darauffolgenden Tage sind die Ringe noch einmal kurz in Süßwasser gespült und danach in Seewasser eingesetzt worden. Das Seewasser in den Boverischalen wurde jeden 2. Tag gewechselt.

Kontrollversuche zeigten, daß bei einer 5 Minuten langen Behandlung der Laichringe mit Sülwasser keine Schädigungen der Eier zu bemerken waren. die Entwicklung verlief normal. Erst nach etwa 1 Woche, nachdem sich die Larven in den Eihüllen drehten, konnten häufiger Anomalien beobachtet werden.

Das Schlüpfen der Veligerlarven geschieht passiv, sie sind nicht imstande, die Eihüllen selbst zu sprengen. Die Haltbarkeit des Mörtelschleimes, der das Sediment im Laichring verkittet, ist begrenzt. Nach einer Zeit, die sich mit der Entwicklungsdauer der nitida-Eier (unter den verschiedenen Temperaturbedingungen) deckt, fallen die Sandkörnchen auseinander, dabei werden die Eihüllen zerrissen, und die Larven gelangen ins freie Wasser.

Versuche, bei denen Embryonen mit Hülle vorzeitig aus dem Ringverband gelöst wurden, bestätigten, daß die Larven, trotz der intensiven Drehbewegung in der Hülle, sich selbst nicht befreien können. Während die Veliger, die sich noch im Laichring befanden, nach dessen Zerfall bereits schlüpften, drehten sich die Larven in den vorher losgelösten Hüllen noch kräftig, als diese nach einigen Tagen schon stark verpilzt waren.

Sämtliche Veliger, die nach der oben beschriebenen Behandlung schlüpften, entsprachen in ihrer Morphologie den Larven, die Thorson für Lunatia nitida beschrieben hat.

\section{Zusammenfassung}

Bei den Untersuchungen über die Funktion der „Bohrdrüse“ der Naticiden konnte zur weiteren Kenntnis der Laichgelege von Lunatia nitida wegen ihrer typischen Form hier "Laichringe" genannt - beigetragen und laichende Weibchen bei der Herstellung der Ringe direkt beobachtet werden.

Nach quantitativen Bodenfaunauntersuchungen von 1949 bis 1960 kommt L. nitida im Ostteil der Deutschen Bucht am häufigsten in schlicksandigen Böden vor.

Die Bestandsschwankungen von Lunatia sind vor allem abhängig von der Abundanz der Lamellibranchier, den Hauptnährtieren der Naticiden. 
Die stark überströmten flacheren Reinsandgründe im Untersuchungsgebiet vor den Nordfriesischen Inseln werden von der kleinen Nabelschnecke gemieden. Die durch die Strömungen verursachten Sandverdriftungen stellen besonders für die auf der Bodenoberfläche liegenden nitida-Laichgelege eine Gefahr dar.

Nach Form und Struktur nehmen die Laichringe von L. nitida eine Sonderstellung unter den bisher beschriebenen Naticiden-Laichgelegen ein. Das wichtigste Maß des Ringes ist der innere Durchmesser, sein Wert deckt sich mit der Gehäusehöhe des entsprechenden Weibchens. Zählungen bei 38 Laichringen ergaben eine durchschnittliche Eizahl von 8140 pro Gelege.

Die Schwierigkeiten, bei $L$. nitida die Geschlechter nach äußeren Schalenmerkmalen zu determinieren, werden besprochen.

Nach Aquariumsbeobachtungen können nitida-Weibchen bis 19 Laichringe in einer Fortpflanzungsperiode ablegen, als Intervall zwischen den einzelnen Laichabgaben wurden im Durchschnitt 4-5 Tage ermittelt. Daraus war zu folgern, daß die Laichzeit am natürlichen Standort 2-6 Monate andauert.

Die Auswahl der Korngröße des im Laichring eingebauten Sandes wird durch "Siebebewegungen" ermöglicht, die der hintere obere Rand des Propodium während des Laichaktes ausführt. Eine Randfalte bewegt sich in etwa $1 \mathrm{sec}$. wellenförmig von rechts nach links. Das auf diese Weise in die unter dem zum Gehäuse hochgezogenen Propodiumteil liegenden Höhlung fallende Sediment wird dort mit Schleim vermischt und, während sich das Weibchen schubweise um das Gehäuse als Achse im entgegengesetzten Uhrzeigersinne dreht, bei jedem Schub auf der rechten Seite herausgedrückt. Dabei gelangen die Eier vom Uterusausgang über die "Eirinne“", einer Hautfaltenduplikatur auf dem Kopffuß, in das Sand-Schleim-Gemisch.

Für 1 Windung des auf diese Weise geformten Laichringes benötigt das Weibchen etwa 2 Stunden. Die um ungefähr 1 Windung vorher abgelegten Eier sind um einen Furchungsschritt voraus.

Die frühesten Furchungsstadien der L. nitida-Eier bis zum ersten Mikromerenquartett werden kurz geschildert und eine Methode, die frisch aus den Aquarien entnommenen Laichringe zum einwandfreien Schlüpfen der Veligerlarven zu bringen, wird beschrieben.

\section{Literaturverzeichnis}

A mio, M., 1955: On the egg masses and larvae of seven species of Naticidae (Gastropoda) from the costal region of Japan: Journ. Shimonoseki Coll. Fish. 5, No. 2.

Ankel. W. E., 1930: Nähreierbildung bei Natica catena (da Costa). Zool. Anz. 89.

- 1936: Prosobranchia. Grimpe-Wagler, Tierwelt der Nord- und Ostsee IX b.

Benthem-Jutting, T. van, 1933: Mollusca (I) A. Gastropoda Prosobranchia et Pulmonata. Fauna van Nederland. Leiden.

Dell, R. K, 1956: Some new off-shore mollusca from New Zealand. Rec. Dominion Mus. $3 / 1$.

Eales, N. B., 1950: The littoral Fauna of Great Britain. A. Handbook for Collectors, at the University Press Cambridge.

Giglioli, M.E.C., 1956: The egg masses of the Naticidae. Fish. Res. Bd. Canada, 12, No. 2.

Mc Ginitie and Nettie Mc Ginitie, 1949; Natural History of marine Animals. Mc GrawHill Book Company, Inc, New York, London, Toronto.

Gravely, F. H, 1942: Shells and other Animal Remains found on the Madras Beach. Bull. Mad. Govt. Mus. (Nat. Hist.) $5(2)$. 
Haller, B., 1893: Die Morphologie der Prosobranchier, gesammelt auf einer Erdumseglung durch die königl. italienische Korvette . Vettor Pisani". Morpholog. Jahrbuch 19.

Hertling, H., 1932: Zur Kenntnis des Laichbandes und der Veligerlarven von Natica pulchella Risso. Zool. Anz, 100, Hft. 3/4.

Hornell, J., 1921: The Common Molluscs of South India. Mad. Fish. Bull. 14.

Jeffreys, J. G., 1867: British Conchology, 4.

Kristensen, I., 1959: The Coastal Waters of the Netherlands as an Environment of Mulluscan Life. Basteria, 23, Suppl.

Lebour, Marie V., 1936: Notes on the Eggs and Larvae of some Plymouth Prosobranchs. Journ. Mar. Biol. Assoc., 20, No. 3, Plymouth.

Linke, O., 1993: Morphologie und Physiologie des Genitalapparates der Nordseelittorinen. Wiss. Meeresunt., Abt. Helgoland, 19.

Morris, P. A., 1950: A Field Guide to the Shells of our Atlantic and Gulf Coasts. Houghton Mifflin Company Boston.

Natarajan, A. V., 1957: Studies on the egg masses and larval development of some prosobranchs from the Gulf of Mannar and the Palk Bay. Proc. Indian Acad. Sci. 46.

Portmann, A., 1925: Der Einfluß der Nähreier auf die Larvenentwicklung von Buccinum und Purpura. Z. Morph. Okol. 3

Schiemenz, P., 1891: Wie bohrt Natica die Muscheln an? Mitt. Zool. Stat. Neapel 10.

Simroth, H., 1896-1907: Gastropoda prosobranchia. Bronn's Klassen und Ordnungen 3, 2.

Thorson, G., 1946: Reproduction and larval development of Danish marine bottom invertebrates. Medd. Komm. Fiskeri- og Havunderseg., Ser. Plankton, 4, No. 1, Kabenhavn.

Ziegelmeier, E., 1954: Beobachtungen über den Nahrungserwerb bei der Naticidae Lunatia nitida Donovan (Gastropoda Prosobranchia). Helg. Wiss. Mecresunt. 5, 1. 